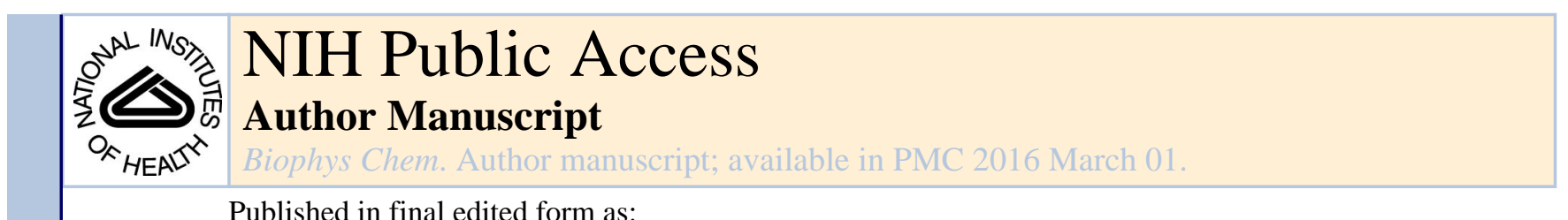

Published in final edited form as:

Biophys Chem. 2015 March ; 198: 22-35. doi:10.1016/j.bpc.2015.01.005.

\title{
Characterization of 3D Voronoi Tessellation Nearest Neighbor Lipid Shells Provides Atomistic Lipid Disruption Profile of Protein Containing Lipid Membranes
}

\author{
Sara Y. Cheng ${ }^{1}$, Hai V. Duong ${ }^{2}$, Campbell Compton ${ }^{2}$, Mark W. Vaughn $^{3}$, Hoa Nguyen $^{4}$, and \\ Kwan H. Cheng 5,6 \\ ${ }^{1}$ Department of Physics, University of Texas at Austin, Austin, Texas 78712, USA \\ ${ }^{2}$ Department of Computer Science, Trinity University, San Antonio, Texas 79212, USA \\ ${ }^{3}$ Department of Chemical Engineering, Texas Tech University, Lubbock, Texas 79409, USA \\ ${ }^{4}$ Department of Mathematics, Trinity University, San Antonio, Texas, 78212 USA \\ ${ }^{5}$ Department of Physics and Astronomy, Trinity University, San Antonio, Texas 78212, USA \\ ${ }^{6}$ Department of Physics, Texas Tech University, Lubbock, Texas 79409, USA
}

\begin{abstract}
Quantifying protein-induced lipid disruptions at the atomistic level is a challenging problem in membrane biophysics. Here we propose a novel 3D Voronoi tessellation nearest-atom-neighbor shell method to classify and characterize lipid domains into discrete concentric lipid shells surrounding membrane proteins in structurally heterogeneous lipid membranes. This method needs only the coordinates of the system and is independent of force fields and simulation conditions. As a proof-of-principle, we use this multiple lipid shell method to analyze the lipid disruption profiles of three simulated membrane systems: phosphatidylcholine, phosphatidylcholine/cholesterol, and beta-amyloid/phosphatidylcholine/cholesterol. We observed different atomic volume disruption mechanisms due to cholesterol and beta-amyloid Additionally, several lipid fractional groups and lipid-interfacial water did not converge to their control values with increasing distance or shell order from the protein. This volume divergent behavior was confirmed by bilayer thickness and chain orientational order calculations. Our method can also be used to analyze high-resolution structural experimental data.
\end{abstract}

\section{(C) 2015 Elsevier B.V. All rights reserved}

Main Correspondence: Sara Y. Cheng, Department of Physics, College of Natural Sciences, University of Texas at Austin, 1 University Station, C1600, Austin, Texas 78212-1192, USA, Phone: (806) 786-3205, sara.y.cheng@utexas.edu. Correspondence Authors: Address all correspondence and reprint requests to Sara Y. Cheng, sara.y.cheng@utexas.edu, Hoa Nguyen, hnguyen5@ trinity.edu or to Kwan H Cheng, biophysics.ttu@gmail.com.

Publisher's Disclaimer: This is a PDF file of an unedited manuscript that has been accepted for publication. As a service to our customers we are providing this early version of the manuscript. The manuscript will undergo copyediting, typesetting, and review of the resulting proof before it is published in its final citable form. Please note that during the production process errors may be discovered which could affect the content, and all legal disclaimers that apply to the journal pertain. 


\section{Keywords}

Voro++; Cholesterol; Lipid Domain; Lipid/Protein Interactions; Annular Lipids

\section{INTRODUCTION}

Protein/lipid interactions play a key role in regulating the structure and function of cell membranes [1-4]. For membrane-active amyloidogenic proteins, a quantitative understanding of the protein-induced lipid disruption behavior upon their insertion into the lipid bilayer provides useful insights into the cytolytic mechanisms of these proteins in cells [5]. Protein-induced lipid disruptions can be quantified experimentally and computationally by examining the structure and dynamics of lipids surrounding the protein [6-10].

Lipids in the presence of a membrane-inserted protein are classified into two classes, annular and non-annular lipids [6]. Annular lipids are those lipids nearest to the inserted protein and non-annular lipids are those that are not. The structural and dynamical properties of these two lipid classes could be compared with those of the bulk lipids. Bulk lipids are lipids in the lipid bilayer of identical composition but in the absence of protein. As expected, the inserted protein perturbs the structure $[6,9,11]$ and dynamics [8] of the annular lipids more than the non-annular and bulk lipids. However, two key questions remain unresolved. First, how do we further classify the non-annular lipids based on their proximity to the annular lipids in a simulation system of limited size? Second, how do we quantify the progressive recovery of the structure of non-annular lipids with increasing distance from the protein at the atomic and molecular level?

Molecular Dynamics (MD) simulations are useful tools to address the above questions on protein-induced disruption on lipids due to the high spatial resolution $(\sim 0.01 \AA)$ and welldefined chemical composition and structure of the protein/lipid membranes. Previous simulation studies on pure lipid bilayers have demonstrated that the time-averaged volume and surface area per lipid, membrane thickness, order parameter, and domain size of the bulk lipids compared favorably with those from experiments [12-21]. In the presence of protein, different analytical tools based on the grid-method [22], Voronoi tessellations (VT) [2], Monte Carlo (MC) Integration [10] and hybrid VT and MC integration [23] have been proposed to study protein/lipid interactions. In most cases, a two-dimensional projection of the protein and lipid atoms or molecules on the bilayer plane was used to classify the annular and non-annular lipids. In addition, most previous studies were focused on the lipid surface area, number density profile, order parameter, and membrane thickness at the whole lipid level. At present, very little is known about the atomistic volume of the perturbed lipids in classified annular and non-annular lipid regions.

In this study, we used a recently established three-dimensional, cell-based VT algorithm (Voro++) $[24,25]$ to classify and characterize protein-induced lipid disruptions in MD simulated lipid bilayers containing large number of atoms with periodic boundaries. Our simulated membrane systems consisted of a model membrane-inserted peptide, betaamyloid $(\mathrm{A} \beta)$, in a fully hydrated binary phosphatidylcholine/cholesterol (PC/CHOL) bilayer and two controls, fully hydrated PC and PC/CHOL bilayers but without protein. The 
use of the VT-derived nearest-atom-neighbor-list of each atom allowed us to classify all the lipids in the $\mathrm{A} \beta / \mathrm{PC} / \mathrm{CHOL}$ bilayer into a single annular lipid shell and multiple non-annular lipid shells. Upon comparing the VT-derived volume of each atom in the lipid shells and the bulk lipids in control bilayers, we explored the volumetric perturbations of CHOL and protein, separately, and examined the recovery behavior of non-annular lipid perturbations at both atomic and molecular levels. Independent biophysical measurements in terms of membrane thickness and lipid orientational order calculations on identical lipid shells were further performed to confirm the VT-based perturbation recovery behavior.

The major goals of this study are to demonstrate that the Voronoi tessellation (VT) - based atomic volumetric distribution analysis of lipid shells is a useful methodology to study lipid/ protein interactions, independent of force fields and simulation conditions, of protein-lipid simulated systems. In addition, the results of the VT analysis in simulated membranes might provide useful insights into revealing the complex lipid disruption mechanisms induced by membrane-active proteins in the crowded environment of biological cell membranes at the single-molecule level. Using previously published data, this study provides a proof-ofconcept of the abilities of this analytical method to validate the cholesterol condensing effect on lipid membranes and provide new insight into the non-uniform effect of protein on the atomic volume and biophysical properties of lipids.

Current interests in membrane biochemistry and biophysics have led to simulations of increasingly complex heterogeneous lipid membranes involving multiple lipids, water, and proteins. Traditional analysis methods often lack sensitivity to clearly distinguish spatial correlations that indicate associations or patterns in nanostructure. As simulation complexity increases, ideas from methods of the mechanics of heterogeneous material in which macroscale properties are predicted from microscale organization and interaction [26] such as the VT analysis will have increased utility. Meso-scale mechanical and electrical properties of the membrane will depend on the geometric arrangement and atomic interaction of membrane components. Techniques like VT provide a foundation to link atomic and mesoscale properties and so will provide clearer physical chemical understanding of the role of membrane structure. Here we demonstrate VT as applied to compute atomic volume, but its usage may also elucidate a host of other properties including meso-scale mechanical processes, including membrane permeability, membrane deformation and perturbation, protein association, and membrane invagination, as few examples.

\section{MATERIALS AND METHODS}

\section{A. Lipid structures and atom types}

Figure 1 shows the schematics of 1-palmitoyl-2-oleoyl-PC and CHOL lipids with each atom labeled by a unique atom type $[15,27]$ from the force fields. Note that all non-polar hydrogen atoms are excluded in the united-atom lipid model [28]. The lipid chemical structures are also illustrated for comparisons. PC is divided into 5 structural regions: choline, phosphate, interface (glycerol backbone, ester oxygen, and carbonyl groups), saturated C16:0 sn-1 chain, and unsaturated 18:1 sn-2 chain. CHOL is grouped into 3 structural regions: polar, four fused rings, and chain. Two distinctive atom groups of PC: 
terminal methyl $\left(\mathrm{CH}_{3}\right.$ groups) of both chains and unsaturated $\mathrm{C}=\mathrm{C}$ of the $s n-2$ chain, are also highlighted. Note that the numbering of atoms and atom names in this study are based on the published protein data bank (PDB) files [27, 29] and do not follow IUPAC-approved convention and nomenclature. A comparison of the IUPAC-atom labeling and the PDB united atom labeling as well as a conversion table are given in Supporting Material, Figure S1 and Table S1.

\section{B. Protein primary structures}

A 40-residue long $\mathrm{A} \beta$ was our model transbilayer protein for investigating protein-induced lipid disruptions in lipid membranes. Its primary sequence [30] is: H-Asp-Ala-Glu-Phe-ArgHis-Asp-Ser-Gly-Tyr-Glu-Val-His-His-Gln-Lys-Leu-Val-Phe-Phe-Ala-Glu-Asp-Val-GlySer-Asn-Lys-Gly-Ala-Ile-Ile-Gly-Leu-Met-Val-Gly-Gly-Val-Val-OH. This protein has two domains: $N$-terminal (Asp1 to Asn27) and $C$-terminal (Lys28 to Val40). A hydrophobic domain spanning Gly29 to Val39 capped by the positively charged Lys28 and negatively charged C-terminus (protonated Val40) is in bold above. At neutral $\mathrm{pH}, \mathrm{A} \beta$ has a net charge of -3 .

\section{MD Simulations}

Three MD simulated membrane systems: $\mathrm{A} / \mathrm{PC} / \mathrm{CHOL}, \mathrm{PC} / \mathrm{CHOL}$, and $\mathrm{PC}$, were analyzed. All simulations were performed in the presence of water. The $\mathrm{A} \beta / \mathrm{PC} / \mathrm{CHOL}$ bilayer comprised one A $\beta, 574 \mathrm{PC}, 383 \mathrm{CHOL}, 211,989$ water and 3 counter $\mathrm{Na}^{+}$ions (initial system size $=13.5 \times 15.6 \times 14.0 \mathrm{~nm}^{3}$ ). The control PC/CHOL bilayer comprised $576 \mathrm{PC}$ and 68,809 water molecules (initial system size $=13.6 \times 14.8 \times 11.6 \mathrm{~nm}^{3}$ ). The control PC bilayer comprised $576 \mathrm{PC}, 384 \mathrm{CHOL}$, and 60,457 water molecules (initial system size = $13.2 \times 14.4 \times 11.7 \mathrm{~nm}^{3}$ ). A $200-n s$ molecular dynamics (MD) simulation with a rectangular, or $x$-, $y$ - and $z-$, periodic boundaries for each bilayer system was performed at 1 atmospheric pressure and a temperature of $300 \mathrm{~K}$ with a 2 fs time step using a modified GROMOS87 and Berger lipid force fields.[29, 31, 32] Detailed procedures and conditions of the atomistic MD simulations of these systems have already been described in detail in our previous study [9]. The Berger force field is a united-atom force field in which $\mathrm{CH}, \mathrm{CH}_{2}$ and $\mathrm{CH}_{3}$ atoms of the $\mathrm{PC}$ and $\mathrm{CHOL}$ are modeled as single methine-, methylene-, or methyl-group particles, as demonstrated in Figure S1 of Supporting Material. For the A $/ \mathrm{PC} / \mathrm{CHOL}$ bilayer used in our analysis, the protein was initially (time $=0 \mathrm{~ns}$ ) in the partially inserted state with $\sim 33 \%$ ahelix in the $N$-terminal and $23 \%$ a-helix in the $C$-terminal. During the $200 \mathrm{~ns}$ simulation time, the protein descended into the bilayer, underwent partial unfolding and ended up in a fully inserted-state with $\sim 22 \%$ alpha-helix, $6 \%$ beta-sheet in the $\mathrm{N}$-terminal and no ordered structure in the $C$-terminal [9]. The equilibrated and stable inserted-state trajectory in the last $30 \mathrm{~ns}$ was analyzed in this VT-based study. This time window was chosen because it is long compared with individual atomic interactions, such as hydrogen bonding [33], but short compared to our simulation time and mesoscale processes such as lipid domain formation. The final simulated structures of protein, water, and lipids in the $\mathrm{A} / \mathrm{PC} / \mathrm{CHOL}$ bilayer are illustrated in Figure 1D. As controls, the last $30 n s$-trajectories of PC and PC/CHOL bilayers under identical simulations conditions were also analyzed using identical algorithms. The trajectories of the three simulation systems and the protein insertion dynamics have been published [9]. The choice of the A $/ \mathrm{PC} / \mathrm{CHOL}$ bilayer for our new proof-of-concept 3D VT 
analysis was based on its large disruptive effect to bulk membrane structures when compared with other simulation systems in our previous non-VT based studies [9, 34].

\section{Three-dimensional Voronoi tessellations of MD simulated membranes}

We employed a three-dimensional Voronoi tessellation computational approach to study protein-induced lipid disruptions. A brief description of the theory underlying this methodology is given here.

Given a set of $N$ particles or generators on a domain $\Omega$, a Voronoi tessellation (VT) divides $\Omega$ into exactly $N$ Voronoi cells. Each Voronoi cell is associated with one particle or a unique generator. Any point inside a given Voronoi cell is closer to its own generator than any other generators. Given a generator $Z_{i}(i=1, \ldots, N)$, its nearest-neighboring generators $Z_{k}(k=1$, $\ldots, N$ and $k \neq i$ ) are those whose Voronoi cells share boundaries with the Voronoi cell of $Z_{i}$. In this pilot study, we used two types of Voronoi diagrams: a regular VT where distance is defined by a Euclidean distance and a radical or power VT whose generator $Z_{i}$ has its own weight $w\left(Z_{i}\right)$. For the latter case, the distance from a point $X$ to the generator $Z_{i}$ is defined as the square of their Euclidean distance minus the square of $\mathrm{w}\left(\mathrm{Z}_{\mathrm{i}}\right)$. More detail about Voronoi tessellations can be found in the surveys [35-37].

We denote a regular VT as non-weighted VT and a radical VT as weighted VT. In our simulations, each atom represents a generator and its van der Waals radius is considered as its weight. Examples of 2D and 3D regular VT-based VT cells are shown in Figures 2A and 2B respectively. In Figure 2A, a two-dimensional (2D) square domain $\Omega$ contains twelve randomly generated particles in black circles $(N=12)$. A $2 \mathrm{D}$ VT method divides this square domain $\Omega$ into twelve Voronoi polygons or $2 \mathrm{D}$ cells with blue boundaries. The generator $Z_{1}$ with its yellow Voronoi region has six nearest neighbors $\left(Z_{2}\right.$ to $\left.Z_{7}\right)$ with different colored Voronoi regions. In Figure 2B, a three-dimensional (3D) cube $\Omega$ contains disperse small particles [25]. A 3D VT method divides this cube domain $\Omega$ into Voronoi polyhedra or 3D cells with flat faces as shown in Figure 2B. In both 2D and 3D regular VT, the line or planes between two nearest particle neighbors form the 2D and 3D bisectors, respectively, of the line joining these two particles. It is important to note that no two Voronoi cells overlap, except for the points on bisectors. So the VT cells span the entire domain $\Omega$, and the sum of the volumes of the VT cells equals to the volume of the $\Omega$.

Rendering of 3D VT cells of the atoms of PC and cholesterol lipids is illustrated in Figures $2 \mathrm{C}$ and 2D, respectively. The color codes are identical to those used in Figures 1A and 1B, respectively. Additionally, the first nearest-neighbor lipids and water of the protein in the A $\beta / \mathrm{PC} / \mathrm{CHOL}$ bilayer based on 3D VT are also demonstrated in Figure 2E.

We employed a recently established 3D VT algorithm (Voro++) [24, 25]. The Voro++ algorithm offers three distinctive features adaptable to our large 3D simulated membrane systems. These features are: (i) Efficient cell-based computations: Voro++ computes each Voronoi cell associated with each generator individually rather than computing the complete Voronoi cells or Voronoi diagram as a single computing object. This approach allows efficient, computation of cell-based statistics, such as cell volume, number of vertices per cell, and nearest-neighbor list, (ii) 3D calculations with periodic boundary conditions: Voro 
++ supports periodic boundary conditions. This feature is important to compute cell-based statistics of any simulated system involving $x$-, $y$-and $z$-periodic boundary conditions as in our simulated membranes, and (iii) weighted and non-weighted features: Voro++ can construct Voronoi diagram with or without van der Waals radii (weight) which allows us to analyze the volume discrepancies in different cases. In this study, two key cell-based statistical parameters, nearest-atom-neighbor list and atomic volume, have been generated for every atom of the simulated membrane at a given time frame of the MD simulation using an in-house Java-based program that incorporates the Voro++ functions on nearest-atomneighbor list and atomistic volume. The program will be available to public after an initial publication of this pilot study. Note that Voro++ has been used in several biophysical and biochemical molecular modeling studies, particularly in micelle systems.[38-41] This work focuses on 3D lipid shell isolation and analysis.

\section{E. Lipid bilayer thickness and segmental orientation order}

The transbilayer, or $z$-dependent, number density profiles of various lipid polar groups were calculated to determine the lipid membrane thickness. Here, a center point along the $z$ direction (bilayer normal) of the upper and lower leaflets was determined. Then, $50 \mathrm{z}$-slices of $1 \AA$ thickness above and $50 \mathrm{z}$-slices of $1 \AA$ thickness below this center point were constructed, for a total of $100,1 \AA z$ slices. The number density in $\mathrm{nm}^{-3}$ of the interested lipid polar group ( $=$ the number of atoms of the selected group inside each $z$-slice divided by the volume of the slice) as a function of the $z$-location of each z-slice was computed at each time frame of the trajectory. Here, the volume of each slice is defined as the height of each slice, $1 \AA$ A, multiplied by the $\mathrm{x}$ and $\mathrm{y}$ dimensions of the simulation box. The peak-to-peak $z$ distance between two well-resolved number density distributions from the lower and upper lipid monolayers was subsequently used to quantify the membrane thickness associated with the selected polar group at each time frame.

An order parameter $\left\langle\mathrm{P}_{2}\right\rangle$ was used to quantify the segmental orientation order of $\mathrm{PC}$ and CHOL [29]. Here $\left\langle\mathrm{P}_{2}\right\rangle$ is defined as $\left(3\left\langle\cos ^{2} \theta\right\rangle-1\right) / 2$. For the $\mathrm{PC}$ acyl chain, $\left\langle\mathrm{P}_{2}\right\rangle$ vs. carbon number of the chain was calculated. Here, $\theta$ is the angle between the $z$-axis and the vector $\left(\boldsymbol{r}_{\mathrm{i}-1}-\boldsymbol{r}_{\mathrm{i}+1}\right)$ joining the nearest-neighbor $(i-1)^{\text {th }}$ and $(i+1)^{\text {th }}$ carbons at $\boldsymbol{r}_{\mathrm{i}-1}$ and $\boldsymbol{r}_{\mathrm{i}+1}$ on each side of the $i^{\text {th }}$ carbon atom at $\boldsymbol{r}_{\mathrm{i}}$. The square bracket denotes the average over all the participating lipids. For $\mathrm{CHOL}$, a single order parameter was determined. Here, $\theta$ is the angle between the $z$-axis and the vector connecting $\mathrm{C} 5$ to $\mathrm{C} 21$, i.e., along the long axis of the sterol rings. All lipid order parameters were calculated using the g-order function of GROMACS [42] at each time frame of the trajectory.

\section{RESULTS}

Time-averaged volumetric and biophysical properties of the lipid bilayers from three membranes, $\mathrm{A} / \mathrm{PC} / \mathrm{CHOL}, \mathrm{PC} / \mathrm{CHOL}$, and PC, over the last $30 \mathrm{~ns}$ (170 to $200 \mathrm{~ns}$ with $1 \mathrm{~ns}$ increment) of the MD trajectories were computed. Each data point represents the mean \pm standard error of the mean (SE), where SE is standard deviation $/ N^{1 / 2}$ with sample size $N=$ 30. We assume that each of the time frames in our analysis is independent of the other frames. The true errors of the calculation are likely to be larger. 


\section{A. Classification of VT shells}

Classification of VT lipid shells refers to the method of sorting lipid molecules into different shells according to their proximity to the inserted protein in the $\mathrm{A} / \mathrm{PC} / \mathrm{CHOL}$ membrane. The nearest-atom-neighbor lists of the protein and lipid atoms calculated from Voro++ [24, 25 ] were used to classify lipids into VT lipid shells. Each shell had a unique shell order $(n)$.

The first-order ( $n=1)$ VT lipid shell consisted of lipid molecules that were nearest neighbors to the protein (see Figure 2E). A nearest neighbor was determined by a lipid selection criterion that at least one atom of the lipid in the first shell must be on the nearestatom-neighbor list of at least one protein atom. For higher-order VT lipid shells $(n \geq 2)$, the selection criterion was that at least one of the lipid atoms of each lipid in the $n^{\text {th }}$ lipid shell must be on the nearest-atom-neighbor list of any lipid atom from the previous $(n-1)^{\text {th }}$ lipid shell. The selection of shells was performed for each time frame.

Both weighted and non-weighted VT methods from Voro++ [24, 25] were used in this work. The classical VT or non-weighted VT neglects atomic radii and only the coordinates of each atom atoms are considered. On the other hand the radical or weighted VT considers the van der Waals radii to weight the atom boundaries according to the relative radii of the atoms.

Figure $3 \mathrm{~A}$ illustrates the lateral view, i.e. along the surface of the planar lipid bilayer in the $x-y$ plane, of all lipid atoms in separated lipid shells at the last time frame (200 ns) of the simulated $\mathrm{A} \beta / \mathrm{PC} / \mathrm{CHOL}$ membrane using weighted VT method. Different orders of VT lipid shells are color-coded in blue ( $1^{\text {st }}$ shell), red ( $2^{\text {nd }}$ shell) black ( $3^{\text {rd }}$ shell), orange $\left(4^{\text {th }}\right.$ shell), yellow ( $5^{\text {th }}$ shell $)$, dark green $\left(6^{\text {th }}\right.$ shell), gray $\left(7^{\text {th }}\right.$ shell $)$ and light green $\left(8^{\text {th }}\right.$ shell) for visualization. Due to the rectangular periodic boundary conditions along the $x$-, $y$ - and $z$ directions in the simulations, the lipids on the simulation box edges were in contact with those on the opposite edge along the same axis. Therefore, four repeating simulation boxes are displayed to reflect these periodic boundary conditions along the $x$ - and $y$-directions. Figure $3 \mathrm{C}$ shows the transverse view, i.e., along the mid $x-z$ cross-section perpendicular to the planar bilayer surface, of the separated lipid shells, using the same color codes as the above lateral view. Only six lipid shells ( $n=1$ to 6 ) are visible. Again, because of the periodic boundary, two repeating simulation boxes along the $x$-direction are demonstrated. Results generated from the non-weighted VT method are shown in Figure S2 of Supporting Material.

Separated VT lipid shells revealed two types of lipid shells, annular and non-annular. The annular lipid shell was the single, first-order $(n=1)$ lipid shell in which all the constituent lipids were nearest to the protein at the single atom level. As shown in Figure 3A, this annular lipid shell exhibited non-uniform surface contour following the transmembrane shape of the protein that came in contact with the lipids. The non-annular lipids were higher-order lipid shells ( $n=2$ to 8 ) surrounding the annular lipids. These non-annular lipid shells could further be separated into two sub-groups depending on the boundary continuity of the lipid shell. As illustrated in Figures 3A and 3C, each lipid shell starting from $n=2$ to 4 formed a continuous, concentric boundary of increasing perimeter around the first annular lipid shell. These shells are defined here as the continuous non-annular lipid group. Due to the finite rectangular size of the simulation box, the remaining higher-order lipid shells ( $n=$ 
5 to 8 ) formed discontinuous, non-concentric, boundaries that filled the interstitial space at the corners of the simulation box. These discontinuous non-annular lipid shells are therefore defined as interstitial non-annular lipids. In this study, we observed 7 or 8 total VT lipid shells over 30 time frames. VT-shell characterization was computed independently for each time frame. Since the last $8^{\text {th }}$ shell was not observed in all 30 frames due to the movement of atoms during simulation, only results from 7 shells were compiled and analyzed.

Water molecules formed nearest neighbors to lipid and protein atoms in our simulated membranes. Here, the nearest water shell, or lipid-interfacial water shell, surrounding each classified VT lipid shell, was also classified. For the lipid-interfacial water classification, the $0^{\text {th }}$ - order $(n=0)$ shell represented those water molecules that were in contact with both protein and lipid atoms. The selection criterion was that at least one of the water atoms in this $0^{\text {th }}$ - order water shell was on the nearest-atom-neighbor lists of any protein and lipid atom. For the higher-order $(n \geq 1)$, the $n^{\text {th }}$ water shell represents the water group in which at least one of the water atoms in that group was on the nearest-atom-neighbor list of any lipid atom of the $n^{\text {th }}$ lipid shell. Figure 3B shows the separated lipid-interfacial water shells ( $n=0$ to 8 ) for the last (200ns) time frame of the simulated $\mathrm{A} \beta / \mathrm{PC} / \mathrm{CHOL}$ membrane. Here, the $0^{\text {th }}$ - order shell is in purple, and the higher order $(n \geq 1)$ shells in identical color-codes as the lipid shells in Figure 3A. It is clear that the lateral distributions of lipid shells and lipidinterfacial water shells are similar suggesting the successfully separation of lipid-interfacial water molecules in our protein-containing membranes.

Careful examinations of the lipid and lipid-interfacial water shells calculated from both weighted and non-weighted methods reveal that almost identical shell classifications were achieved in both methods except those at high orders (see Figure S2 in Supporting Material). Volumetric and biophysical characterizations of these shells from both methods are given below.

\section{B. Volumetric properties of lipid bilayers}

The atomic volumes of lipid and lipid-interfacial water in control and protein-containing bilayers were determined. We focused on analyzing separately the volume perturbation effects of CHOL on PC bilayers and that of the inserted protein $\mathrm{A} \beta$ on PC/CHOL bilayers.

\section{Volume perturbation effects of cholesterol on PC bilayers-Time-averaged} volumes of individual atoms in PC and PC/CHOL bilayers without protein (control membranes) were calculated separately using the weighted-VT method, which takes into account the van der Waals radius. As demonstrated in the first column of Figure 4, the timeaveraged volume of the lipid atom vs. atom type along the length of the lipid, i.e., from the polar headgroup to the hydrophobic tail, varied over a broad range. In PC, a volume of 6-7 $\AA^{3}$ for the N4 or P8 atom in the headgroup (Figure 4A) to a volume of $40-45 \AA^{3}$ for the terminal methyl carbon atom (C50 or CA2) in the chain (Figures $4 \mathrm{E}$ and $4 \mathrm{G}$ ) was found. This large volume variation reflects the differences in the bonding and non-bonding environments among different united atoms in the lipid. Referring to Figure 1A, the polar, $\mathrm{N} 4$ or P8 atom is covalently bonded to four neighboring united atoms in the PC headgroup, while the non-polar, teminal methyl C50 or CA2 united atom is covalently bonded to only 
one neighboring chain united atom. Therefore, the terminal united atom has a "larger" free space than the tetravalent atom in PC. In CHOL, similar observations of larger volume in monovalent united atoms than in multivalent atoms, e.g., C1, C17, and C23 of $\sim 35 \AA^{3}$ vs. C2 and $\mathrm{C} 16$ of $\sim 5 \AA^{3}$, were evident as shown in Figure 4I. Similar atomic volume profile was found in both PC and CHOL using non-weighted VT method (Figure S2 of Supporting Material).

To assess the time-averaged volume perturbation effect of $\mathrm{CHOL}$ on $\mathrm{PC}$ in the $\mathrm{PC} / \mathrm{CHOL}$ bilayer at the atomic level, the volume difference, defined as the time-averaged volume of each atom in the PC/CHOL bilayer minus that in the PC bilayer, was computed. As shown in the second column of Figure 4, the time-averaged atomic volume difference vs. atom type plot provides a quantitative measurement of the volumetric perturbation of $\mathrm{CHOL}$ along the length of PC with single atom resolution.

In the PC headgroup region (Figure 4B), we observed a small volume enhancement (positive) effect of CHOL of $+0.1 \AA^{3}$ on the $\mathrm{C} 5$ atom of the 6 -atom choline group but a larger volume condensing (negative) effect of $\mathrm{CHOL}$ of -0.2 to $-0.5 \AA^{3}$ on the $\mathrm{O} 7, \mathrm{O} 9$, and $\mathrm{O} 11$ atoms of the 5-atom phosphate group. In the interface region (Figure 4D), except for a small positive CHOL effect of $+0.2 \AA^{3}$ on the $\mathrm{C} 12$ atom of glycerol, we identified a predominantly negative CHOL effect, particularly of -0.5 and $-1 \AA^{3}$ on the $\mathrm{O} 35$ and $\mathrm{O} 14$ atoms, respectively, for this 9-atom interface group. Large and exclusively negative $\mathrm{CHOL}$ effects were found in the 15- and 17-atom acyl chain regions of PC. As shown in Figure 4F, a well-defined dip or large negative peak of $\sim-2 \AA^{3}$ at the $\mathrm{C} 43$ atom located at the middle of the PC saturated $s n-1$ (C16:0) chain and a rising negative edge of similar value at the terminal C50 atom were evident. In contrast, poorly defined "dip and edge" features were found in the unsaturated $s n-2$ (C18:1) chain as shown in Figure 4H. Interestingly, a close inspection of the "spectrum-like" volume difference vs. atom type plot in Figure $4 \mathrm{H}$ reveals an interesting "symmetry" of the volume difference with respect to the middle of the $s n-2$ chain, i.e., between $\mathrm{C} 24$ and $\mathrm{C} 25(\mathrm{C}=\mathrm{C})$. Here, the volume difference profile from $\mathrm{C} 24$ towards $\mathrm{C} 17$ is almost identical to that from $\mathrm{C} 25$ to $\mathrm{CA} 1$ along the length of the $s n-2$ chain in opposite directions, respectively.

In order to compare the $\mathrm{CHOL}$ perturbation effects among different regions of $\mathrm{PC}$, the timeaveraged volume difference per atom in each region of $\mathrm{PC}$ was calculated, and the results were $0.02 \pm 0.01,-0.20 \pm 0.01,-0.39 \pm 0.01,-1.40 \pm 0.02$, and $-1.35 \pm 0.02 \AA^{3} /$ atom for the choline, phosphate, interface, $s n-1$ chain and $s n-2$ chain, respectively. Here, a progressive atomic volume condensing effect along the PC lipid from head to tail was evident.

Therefore, the commonly known cholesterol condensing effect, manifested as the reduction of $\mathrm{PC}$ atomic volume by $\mathrm{CHOL}$, was much stronger in the hydrocarbon chain region than in the headgroup or interface region. Also, the above "volume difference spectrum" of the $s n-1$ chain suggests a regionally stronger $\mathrm{CHOL}$ effect is at the middle of saturated chain and the terminal methyl group of PC.

Similar volume difference profile was also detected using the non-weighted VT methods as shown in Figure S3 of Supporting Material. 
2 Volume perturbation effects of protein on PC/CHOL bilayers-The effects of a transmembrane protein $\mathrm{A} \beta$ on the time-averaged volumetric properties of both $\mathrm{PC}$ and CHOL lipid molecules in separated VT lipid shells ( $n=1$ to 7 ) at the atomic level have been investigated. Similar to the approach of investigating the perturbation effects of CHOL on $\mathrm{PC}$ atoms, the atomic volume difference, defined as the volume of each atom in the $\mathrm{A} \beta / \mathrm{PC} /$ CHOL bilayer minus that in the control PC/CHOL bilayer, was computed.

The volume difference vs. atom type profiles or "spectra" for the annular and non-annular lipids are presented in the second column of Figure 4 and in Figures S4, S6, and S8 of Supporting Material based on the weighted-VT method. The corresponding plots for the non-weighted method are given in the second column of Figure S3 and in Figures S5, S7, and S9 of Supporting Material. For all lipid shells, very similar positive and negative volume differences among lipid atoms were observed in both weighted and non-weighted VT calculations.

Positive volume differences were detected in 16 out of $52 \mathrm{PC}$ atoms, mostly in the first half of the acyl chains (Figures $4 \mathrm{~F}$ and $\mathrm{H}$ ), and in 13 out of $29 \mathrm{CHOL}$ atoms (Figure $4 \mathrm{~J}$ ). In contrast, small positive volume differences for the CHOL-condensing effect were found in only 2 atoms, i.e., C5 in the choline (Figure 4B) and C12 in the glycerol (Figure 4D) of PC. These results suggest a different volume perturbation mechanism of transmembrane protein on its nearby annular PC atoms than the predominant condensing effect of cholesterol on the bulk PC atoms.

In order to compare the protein perturbation effects among different regions of $\mathrm{PC}$, the timeaveraged volume difference per atom in each region of PC in the annular lipid shell was calculated, and the results were $-0.08 \pm 0.04,-0.13 \pm 0.03,-0.21 \pm 0.03,-0.31 \pm 0.04$ and $-0.14 \pm 0.04 \AA^{3} /$ atom for the choline, phosphate, interface, $s n-1$ chain, and $s n-2$ chain, respectively. Except the choline group, the volume condensing effect of the transmembrane protein on PC was significantly smaller than that of CHOL on PC (see above). For the PC choline group, the small positive volume difference of $0.02 \AA^{3}$ from the cholesterol is strikingly different from the small, negative volume difference of $-0.08 \AA^{3}$ from the transmembrane protein. Therefore, the transmembrane protein reduces the average atomic volume of all regional groups with the strongest effect on the saturated $s n-1$ chain region of PC as opposed to the progressive negative volume effect of cholesterol from the headgroup to the tail regions with similar influences on both chains.

For the non-annular ( $n>1$ ) PC lipids (Figures S4-S9 of Supporting Material), a general decrease in the atomic volume difference with increasing $n$ was evident till the $4^{\text {th }}$ or $5^{\text {th }}$ order. However, the volume difference reappeared as $n$ increased from $5^{\text {th }}$ to $7^{\text {th }}$ for both weighted and non-weighted VT.

The perturbation effects of membrane protein on the regional group, or fractional, volume of PC were investigated. Figure 5 shows the time-averaged fractional lipid volume vs. $n$ plot for each regional lipid group of PC for both weighted (in red) and non-weighted (in black) calculations. The fractional lipid volume of the bulk PC lipids in the control PC/CHOL bilayer is shown for comparison. It is clear that a convergence of the fractional lipid volume 
to the control volume occurred at $n=2$ and 4 for the choline, $n=3$ and 6 for the phosphate, $n=3-7$ for the interface, $n=4,6$, and 7 for the $s n-1$ chain and $n=4$ for the $s n-2$ chain. Interestingly, the $\mathrm{C}=\mathrm{C}$ of $s n-2$ chain started from a volume higher than the control and converged to the control at $n=3,4$, and 6 . On the other hand, the terminal $\mathrm{CH}_{3}$ of both chains started from lower than the control and converged to the control at $n=4$. These highly heterogeneous, or regional dependent, perturbation effects across different orders of non-annular lipid shells suggest a non-progressive effect of membrane protein on the atomic volume of different regional groups of PC. The above trend was also found in non-weighted VT. Interestingly, the PC-choline volume from weighted VT was significantly higher than that from non-weighted VT for each shell. Yet, negligible differences between weighted and non-weighted VT volumes were noticed in other regional groups.

Finally, the perturbation effect of protein on the whole molecule volume of PC was examined. As shown in Figure 6C, an interesting "over-damping" behavior in which the PC volume oscillated around the control value before converging at $n=6$ and 7 in the interstitial lipid domain was evident.

Similar analysis of the volume perturbation effects of protein on CHOL was performed. At the atomic level, predominantly positive or volume enhancing effects of protein on CHOL was evident in the annular shell $(n=1)$ as shown in Figure 4J. As shown in Supporting Material, this mostly positive volume difference effect diminished at $n=4$ but reappeared at higher order. At the regional group level (Figures $5 \mathrm{G}-\mathrm{I})$, a very small $\left(<1 \AA^{3}\right)$ oscillatory behavior of volume around the control was observed for the polar group. On the other hand, an abrupt decline of the rings volume from $n=1$ to 4 until it reached the control following by a small overshoot of volume at higher orders, and finally, a sigmoidal drop behavior from a higher value to the control was found for the chain region. At the whole molecule level (Figure 6D), a clear sigmoidal behavior with convergence from a higher volume value to lower control value after the $4^{\text {th }}$ order was clearly found. Identical behavior was detected in non-weighted VT.

Other than PC and CHOL lipid volumes, the perturbation effects of protein on the lipidinterfacial water volume in each lipid shell were also studied as shown in Figure 6B. Here a drop of the lipid-interfacial water volume from $34 \AA^{3}$ in the immediate vicinity of the protein $(n=0)$ to a lower value of $33 \AA^{3}$ at the highest order shell $(n=7)$ was found. Good agreement with the control value at $n=3$ to 5 was evident. The lipid-interfacial water volume in weighted VT was slightly smaller than in non-weighted VT. However, the difference was insignificant.

To assess the effect of the membrane protein on the compositions of lipid and lipidinterfacial water in VT shells, time-averaged counts of PC, CHOL, and lipid-interfacial water molecules of each VT shell were determined and are shown in Figure 6A. Weighted and non-weighted calculations show identical results. The number of lipids and lipidinterfacial water increased progressively with $n$ until the $5^{\text {th }}$ order and started to decline at higher order. A plot of CHOL mole\% (insert of Figure 6A) revealed that the cholesterol content was $\sim 26 \%$ in the annular lipid region $(n=1)$, reached a plateau of $\sim 40 \%$ for $n=2$ to 
5 and increased to $46 \%$ as $n$ increased further to 7 . The bulk CHOL mole $\%$ concentration of our $\mathrm{A} \beta / \mathrm{PC} / \mathrm{CHOL}$ membrane was $40 \%$.

\section{Bilayer thickness and order parameter in lipid shells}

Bilayer thicknesses of different lipid polar groups were determined from the time-averaged peak-to-peak distances of the number density vs. $z$ plots. The first column of Figure 7 illustrates the number density vs. z plots of PC-phosphate, PC-carbonyl, and CHOL-O6 in the $\mathrm{A} \beta / \mathrm{PC} / \mathrm{CHOL}$ (annular shell), $\mathrm{PC} / \mathrm{CHOL}$ and PC bilayers based on weighted VT.

The time-averaged peak-to-peak distances of these lipid polar groups from all lipid shells ( $n$ $=1$ to 7 ) as well as from the controls ( $\mathrm{PC}$ and $\mathrm{PC} / \mathrm{CHOL}$ bilayers) were computed and are presented in the second column of Figure 7. For the controls, the thicknesses of PCphosphate (Figure 7B) and PC-carbonyl (Figure 7D) started from 36 and 30 in the control PC bilayer, but increased to 44 and $35 \AA$ in the control PC/CHOL bilayer. These results support the volume condensing effect of CHOL on PC in terms of an increase in the thickness of the bilayer.

For the lipid shells, the thickness of PC-phosphate increased progressively from $n=1$ to 4 , reached the control value but declined at higher orders as shown in Figure 7B. The thickness of PC-carbonyl followed similar trend of converging from $n=1$ to 4 , decreased at higher orders of $n=5$ and 6, but converged back to the control again at $n=7$ (Figure 7D). Interestingly, the thickness of CHOL-O6 exhibited a complete convergence behavior with increasing $n$ as shown in Figure 7F. Here its value reached the control at $n=3$ and remained constant at all higher $n$ values.

The time-averaged order parameters of $\mathrm{PC}$ and $\mathrm{CHOL}$ are shown in Figure 8. Figures 8A and $8 \mathrm{~B}$ show the PC chain order parameter vs. carbon number of the $s n-1$ and $s n-2$ chains, respectively, in the annual lipid region $(n=1)$ of the $\mathrm{A} \beta / \mathrm{PC} / \mathrm{CHOL}$ and in the bulk lipids of the control PC and PC/CHOL bilayers. As expected, $\mathrm{CHOL}$ produced a significant increase in the PC chain order parameters of both sn- 1 and sn-2 acyl chains in the PC/CHOL bilayer when compared with those in the PC bilayer. The chain order parameter of the annular lipids was much lower than that of the control PC/CHOL bulk lipids but still higher than that of the control PC bulk lipids. The PC chain order parameter difference, defined as the PC chain order parameter in $\mathrm{A} / \mathrm{PC} / \mathrm{CHOL}$ minus the $\mathrm{PC}$ chain order parameter in $\mathrm{PC} / \mathrm{CHOL}$, vs. lipid shell number plots are given in Figure $8 \mathrm{C}$ and Figure $8 \mathrm{D}$ for $s n-1$ chain and $s n-2$ chain, respectively. Here, we observed a recovery of the $\mathrm{PC}$ chain order parameter of either the $s n-1$ or $s n-2$ chain from $n=1$ to 4 towards the control and then a significant deviation from the control for higher orders. Interestingly, the sterol ring order parameter of CHOL (insert of Figure 8B) revealed a similar recovery of the ring order toward the control value from $n=$ 1 to 4 and a deviation form the control at higher $n$.

Bilayer thickness and order parameter were also calculated using non-weighted VT as shown in Figures S10 and S11 of Supporting Material. They agreed with those from weighted VT within the uncertainty of each data point. 


\section{DISCUSSION}

A new 3D VT-based nearest-atom-neighbor method was used to classify lipid molecules into shells of increasing order according on their proximity to a membrane protein in threeor $x y z$ dimensions. Previous MC-, VT- and grid-based studies on lipid domains and lipid/ protein interactions $[9,13,22,23]$ projected the atoms or the center-of-masses of lipids on the bilayer surface or $x y$-plane. This projection approach might introduce uncertainties in the lipid classification and averages out atomistic detail about the structure of the protein-lipid bilayer interactions. Due to the computational efficiency and accuracy of the cell-based Voro ++ [25], exact nearest-atom-neighbors separated by common Voronoi faces were identified for all lipids without the use of projection or any assumptions. Therefore, the first or annular lipid shell comprised lipid molecules closest to the membrane protein at the single-atom level. Similarly, any higher order $(n>1)$ lipid shell, or non-annular lipid shell, contained lipids adjacent to the lipids of the previous shell, also at the atomic level. Weighted and nonweighted VT produced similar results in both classification of lipid shells and characterization of volume and biophysical properties among those shells.

Some previous analyses of membrane simulations required assumptions about molecular size or shape taken from experimental data $[14,16,28]$ or forces between neighboring atoms [43]. The technique presented here depends only on the parameters of the simulation, and therefore are internally consistent. The xyz-coordinates of the atoms in the simulation box at a given time frame were the only input parameters of our shell computation. Therefore, this three-dimensional, atomistic lipid shell classification method is very general in nature and can be applied to any simulated membrane systems with no specific force field requirements or assumptions of the structure or shape of the lipid component. Of course, the results of the analysis depend on the specific force fields used. In addition, since the only input is the coordinates of the particles, this 3D VT shell method can readily be applied to analyze membrane disruptive profile of structural experimental data, e.g., x-ray and NMR.

Due to the intrinsic and necessary periodic boundaries in membrane simulations, we further classified the non-annular lipids $(n>1)$ into shells of continuous boundaries $(1<\mathrm{n}<5)$ and shells of non-continuous boundaries $(n>5)$. The latter represent interstitial lipids that occupied the corners of the rectangular simulation box. These interstitial lipids were affected by not just one inserted protein but by four nearest self-replicating inserted proteins. The use of Voro++ that supports periodic boundaries allowed us to unequivocally identify these interstitial lipids from the continuous non-annular lipids. We propose that the approach of separating lipids into annular, non-annular, and interstitial-non-annular lipid domains provides a quantitative and approximation-free means to examine protein-induced deformation zones in lipid membranes based on their proximity from the replicating proteins in simulations. The influence of lipids by multiple proteins also reflects the compositionally heterogeneous and highly crowded environment of biological membranes $[4,11]$.

Detailed characterizations of the volume perturbations of phospholipid by cholesterol and membrane protein represent an ongoing challenge in studying the structure-function relationship of lipid membranes. For a one-component PC bilayer, the molecular volume of PC has been measured using neutral flotation and x-ray measurements $[17,18]$ that provides 
an important structural parameter for the validation of force fields in membrane simulations [19, 20, 43-48]. For a two-component PC/CHOL lipid bilayer, the partial molecular volume of each lipid has been determined from the volume per molecule vs. cholesterol mole fraction plot generated from experiments [16]. Assumptions about the lipid-associated water, a fixed volume of $\mathrm{CHOL}$, and the cylindrical shape of the lipid molecule are required $[14,16-18,28]$. Using the same PC/CHOL bilayer as in this study, the molecular volumes of PC and CHOL were found to be 1256 and $623 \AA^{3}$, respectively, from a previous experiment [17]. These values were compared with the VT volume of 1127 and $602 \AA^{3}$, respectively, from this simulation study in which lipid-associated water molecules $\left(\sim 31 \AA^{3 /}\right.$ molecule) were explicitly separated from the lipids. A difference of $\sim$ four or one lipidassociated water for $\mathrm{PC}$ or $\mathrm{CHOL}$, respectively, may explain the discrepancy between the experiment and simulation results other than the choice of force fields in simulations. Note that absolute volume values from VT depends on the force fields. An accurate calculation of atomistic and regional volume from the method will allow us to validate or improve force field parameters in membrane biophysics.

We observed a feature rich spectrum-like volume difference vs. atom type plot that indicated a localized volume perturbation effect of $\mathrm{CHOL}$ and protein. For the perturbation by cholesterol, a dip or a symmetric distribution around the central atom of the $s n-1$ or $s n-2$ acyl chains of the PC revealed that the single double bond at the center of the sn-2 acyl chain created significant perturbation that made the middle of both acyl chains the soft, or highly compressible region of the lipid bilayer. The volume difference at the middle of the chain was nearly identical to the highly flexible and compressible terminal methyl ends of the chains. Interestingly, the presence of a transmembrane protein revealed a transition from a positive volume to a negative volume effect at the middle of the acyl chain as well. This further supports the significance of a single double bond in the acyl chain in modulating the volume perturbation effects of $\mathrm{CHOL}$ and membrane protein in the lipid bilayer. Note, that the volume condensing effect of $\mathrm{CHOL}$ on $\mathrm{PC}$ has been found in a previous calorimetric measurement [49] on the same PC/CHOL bilayer.

We observed a stronger volume condensing effect of $\mathrm{CHOL}$ and protein on the non-polar acyl chain region than the polar (headgroup and interface) region of PC. This observation indicates a large difference in the volume compressibility of the polar and hydrophobic regions of a PC lipid. The reason why the polar region of PC was less perturbed by $\mathrm{CHOL}$ or protein might be associated with the presence of strong hydrogen bonding and coulomb interactions among the polar and charged atoms in the polar region. These interactions make the packing density of atoms in the polar region less compressible than the acyl chain region. The latter involve steric and hydrophobic interactions among atoms. Previous experimental [16, 49-51] and simulation studies [12, 14, 28, 52] indicated the condensing effect of cholesterol on PC is mainly limited to the non-polar cholesterol ring region and therefore support our findings here.

Our results revealed an intriguing non-uniform recovery behavior of lipid volumes to the control values with increasing shell order number. The total volume of either PC or CHOL converged towards the bulk lipid value with increasing lipid shell order. This suggests that interstitial lipids ( $n=6$ and 7 ) at the whole volume level behave like the bulk lipids at the 
whole lipid level. However, some fractional volumes of both PC and CHOL converged to the bulk lipid value within the concentric non-annular lipids ( $n=4$ or 5 ) but diverged from the bulk lipid value at higher shell order or within the interstitial lipids. These results provide evidence that the volumetric properties of the interstitial lipids did not fully recover to the bulk lipid properties. The divergent behavior of the lipid-interfacial water volume as well as the independent membrane thickness and lipid orientational order measurements further support the divergent behavior of the volume of interstitial lipids. Therefore the volumetric properties of lipids are highly heterogeneous reflecting a long-range perturbation effect of inserted protein up to several lipid shells away from the first annular shell. Note that the presence of interstitial lipids is directly related to the limited system size in any MD simulations involving periodic boundaries. The perturbation in those interstitial lipids will be stronger in smaller simulation systems.

Lipid-peptide interactions are characterized by force-field parameters. For the GROMOS/ Berger lipids used here, it should be noted that the lipid-peptide interactions are likely overestimated. Kukol [53] found that the secondary structure and integrity of a transmembrane protein was similar for both the GROMOS/Berger force field and the frequently used GROMOS96 53A6 field, but after $7 \mathrm{~ns}$ of simulation, the protein in the GROMOS/Berger field did show greater root mean square deviation, suggesting the structure was somewhat less stable. Tieleman et al. [43] found that the strength of lipidprotein interaction appeared to be overestimated by the combination of the GROMOS/ Berger field. In this study, overestimated lipid-protein interaction strength would show reduced lipid volume at low shell number, however, the amount of reduction has not been determined yet. The sensitivity of the VT method in determining the lipid volume may provide valuable information in quantifying the performance of various force fields in describing lipid/cholesterol/protein interactions.

The composition profile among lipid shells revealed an interesting lateral cholesterol distribution behavior. The concentration of cholesterol was lowest in the annular lipid shell but highest in the interstitial-non-annular lipid shell. The concentric non-annular lipid shell had similar cholesterol concentration like the bulk lipids in PC/CHOL. These results indicate a complex distribution of cholesterol into varying concentration zones, low, medium, and high, surrounding a membrane protein in our simulation system. Longer simulation time is needed to observe redistribution of cholesterol due to diffusion in the presence of protein.

\section{CONCLUSIONS}

Using a novel 3D VT computational approach, lipids were classified into shells based on the proximity of the lipids to an inserted protein in a simulated lipid membrane of limited size. We distinguish up to eight lipid shells around a beta-amyloid peptide, analyze the lipid order, membrane thickness, and volume effects. Weighted and non-weighted calculations on volume are reported and validated with earlier studies on the effect of cholesterol. The observed complex volume perturbation profile of the lipid shells suggests heterogeneous volume distribution of lipids surrounding the protein in a compositionally and structurally heterogeneous lipid bilayer, reflecting the crowded molecular environment of cell membranes. Our VT-based lipid shell analysis methodology can be generalized and applied 
to study lipid/protein interactions of simulated membranes independent of the simulation conditions and force fields as well as structural experiment data of membranes.

\section{Supplementary Material}

Refer to Web version on PubMed Central for supplementary material.

\section{ACKNOWLEDEMENT}

This work was supported by the Robert A. Welch Research Foundation grant (D-1158), NIH grant (GM090897-02), Williams Endowment fund of Trinity University, Texas Advanced Computing Center (TACC) for the use of Lonestar Cluster under the project (G-803132) "Protein Unfolding in Lipid Membranes", and Texas Tech University Supercomputing Center for supporting in computing. The authors would like to acknowledge the valuable help of the developer of Voro++, Dr. Chris Rycroft, Applied Mathematics, School of Engineering and Applied Sciences, Harvard University, for his insightful discussions about Voro++ and advice about treatment of periodic boundary conditions in our code used in this study.

\section{REFERENCES}

1. Anderson RG, Jacobson K. A role for lipid shells in targeting proteins to caveolae, rafts, and other lipid domains. Science. 2002; 296:1821-1825. [PubMed: 12052946]

2. Hildebrand PW, Rother K, Goede A, Preissner R, Frommel C. Molecular packing and packing defects in helical membrane proteins. Biophys. J. 2005; 88:1970-1977. [PubMed: 15556989]

3. Lee AG. Biological membranes: the importance of molecular detail. Trends Biochem. Sci. 2011; 36:493-500. [PubMed: 21855348]

4. Phillips R, Ursell T, Wiggins P, Sens P. Emerging roles for lipids in shaping membrane-protein function. Nature. 2009; 459:379-385. [PubMed: 19458714]

5. Butterfield SM, Lashuel HA. Amyloidogenic protein-membrane interactions: mechanistic insight from model systems. Angew. Chem. Int. Ed. 2010; 49:5628-5654.

6. Lee AG. How lipids affect the activities of integral membrane proteins. Biochim. Biophys. Acta. 2004; 1666:62-87. [PubMed: 15519309]

7. Lemkul JA, Bevan DR. Lipid composition influences the release of Alzheimer's amyloid betapeptide from membranes. Protein Sci. 2011; 20:1530-1545. [PubMed: 21692120]

8. Niemela PS, Miettinen MS, Monticelli L, Hammaren H, Bjelkmar P, Murtola T, Lindahl E, Vattulainen I. Membrane proteins diffuse as dynamic complexes with lipids. J. Am. Chem. Soc. 2010; 132:7574-7575. [PubMed: 20469857]

9. Qiu L, Buie C, Reay A, Vaughn MW, Cheng KH. Molecular dynamics simulations reveal the protective role of cholesterol in beta-amyloid protein-induced membrane disruptions in neuronal membrane mimics. J. Phys. Chem. B. 2011; 115:9795-9812. [PubMed: 21740063]

10. Qiu L, Lewis A, Como J, Vaughn MW, Huang J, Somerharju P, Virtanen J, Cheng KH. Cholesterol modulates the interaction of beta-amyloid peptide with lipid bilayers. Biophys. J. 2009; 96:4299-4307. [PubMed: 19450500]

11. Aponte-Santamaria C, Briones R, Schenk AD, Walz T, de Groot BL. Molecular driving forces defining lipid positions around aquaporin-0. Proc. Natl. Acad. Sci. U.S.A. 2012; 109:9887-9892. [PubMed: 22679286]

12. Martinez-Seara H, Rog T, Karttunen M, Vattulainen I, Reigada R. Cholesterol induces specific spatial and orientational order in cholesterol/phospholipid membranes. PloS one. 2010; 5:e11162. [PubMed: 20567600]

13. Pandit SA, Vasudevan S, Chiu SW, Mashl RJ, Jakobsson E, Scott HL. Sphingomyelin-cholesterol domains in phospholipid membranes: atomistic simulation. Biophys. J. 2004; 87:1092-1100. [PubMed: 15298913]

14. Alwarawrah M, Dai J, Huang J. A molecular view of the cholesterol condensing effect in DOPC lipid bilayers. J. Phys. Chem. B. 2010; 114:7516-7523. [PubMed: 20469902] 
15. Zhu Q, Cheng KH, Vaughn MW. Molecular dynamics studies of the molecular structure and interactions of cholesterol superlattices and random domains in an unsaturated phosphatidylcholine bilayer membrane. J. Phys. Chem. B. 2007; 111:11021-11031. [PubMed: 17718554]

16. Edholm O, Nagle JF. Areas of molecules in membranes consisting of mixtures. Biophys. J. 2005; 89:1827-1832. [PubMed: 15994905]

17. Greenwood AI, Tristram-Nagle S, Nagle JF. Partial molecular volumes of lipids and cholesterol. Chem. Phys. Lipids. 2006; 143:1-10. [PubMed: 16737691]

18. Kucerka N, Tristram-Nagle S, Nagle JF. Structure of fully hydrated fluid phase lipid bilayers with monounsaturated chains. J. Membr. Biol. 2005; 208:193-202. [PubMed: 16604469]

19. Benz RW, Castro-Roman F, Tobias DJ, White SH. Experimental validation of molecular dynamics simulations of lipid bilayers: a new approach. Biophys. J. 2005; 88:805-817. [PubMed: 15533925]

20. Klauda JB, Kucerka N, Brooks BR, Pastor RW, Nagle JF. Simulation-based methods for interpreting X-ray data from lipid bilayers. Biophys. J. 2006; 90:2796-2807. [PubMed: 16443652]

21. Hassan-Zadeh E, Baykal-Caglar E, Alwarawrah M, Huang J. Complex roles of hybrid lipids in the composition, order, and size of lipid membrane domains. Langmuir. 2014; 30:1361-1369. [PubMed: 24456489]

22. Allen WJ, Lemkul JA, Bevan DR. GridMAT-MD: a grid-based membrane analysis tool for use with molecular dynamics. J. Comput. Chem. 2009; 30:1952-1958. [PubMed: 19090582]

23. Mori T, Ogushi F, Sugita Y. Analysis of lipid surface area in protein-membrane systems combining voronoi tessellation and monte carlo integration methods. J. Comput. Chem. 2012; 33:286-293. [PubMed: 22102317]

24. Rycroft, CH. Voro++: A Three-Dimensional Voronoi Cell Library in C++. Berkeley, CA: Lawrence Berkeley National Laboratory; 2009. Available at http://math.lbl.gov/voro++/.

25. Rycroft CH. VORO++: a three-dimensional voronoi cell library in C++ Chaos. 2009; 19:041111. [PubMed: 20059195]

26. Torquato, S. Random Heterogeneous Materials: Microstructure and Macroscopic Properties. New York: Springer-Verlag; 2002.

27. Holtje M, Forster T, Brandt B, Engels T, von Rybinski W, Holtje HD. Molecular dynamics simulations of stratum corneum lipid models: fatty acids and cholesterol. Biochim. Biophys. Acta. 2001; 1511:156-167. [PubMed: 11248214]

28. Hofsass C, Lindahl E, Edholm O. Molecular dynamics simulations of phospholipid bilayers with cholesterol. Biophys. J. 2003; 84:2192-2206. [PubMed: 12668428]

29. Berger O, Edholm O, Jahnig F. Molecular dynamics simulations of a fluid bilayer of dipalmitoylphosphatidylcholine at full hydration, constant pressure, and constant temperature. Biophys. J. 1997; 72:2002-2013. [PubMed: 9129804]

30. Selkoe DJ. The molecular pathology of Alzheimer's disease. Neuron. 1991; 6:487-498. [PubMed: 1673054]

31. van Buuren AR, Marrink SJ, Berendsen HJC. A molecular dynamics study of the decane/water interface. J. Phys. Chem. 1993; 97:9206-9212.

32. van der Spoel D, van Buuren AR, Tieleman DP, Berendsen HJC. Molecular dynamics simulations of peptides from BPTI: a closer look at amide-aromatic interactions. J. Biomol. NMR. 1996; 8:229-238. [PubMed: 8953214]

33. Chanda J, Chakraborty S, Bandyopadhyay S. Sensitivity of hydrogen bond lifetime dynamics to the presence of ethanol at the interface of a phospholipid bilayer. J. Phys. Chem. B. 2006; 110:3791-3797. [PubMed: 16494438]

34. Qiu L, Buie C, Cheng KH, Vaughn MW. Scaling and alpha-helix regulation of protein relaxation in a lipid bilayer. J. Chem. Phys. 2014; 141:225101. [PubMed: 25494768]

35. Aurenhammer F. Voronoi diagrams - a survey of a fundamental geometric data structure. ACM Comput. Surv. 1991; 23:345-405.

36. Poupon A. Voronoi and Voronoi-related tessellations in studies of protein structure and interaction. Curr. Opin. Struct. Biol. 2004; 14:233-241. [PubMed: 15093839]

37. Aurenhammer F. Power diagrams: properties, algorithms and applications. SlAM J. Comput. 1987 
38. Allen DT, Saaka Y, Lawrence MJ, Lorenz CD. Atomistic description of the solubilisation of testosterone propionate in a sodium dodecyl sulfate micelle. J. Phys. Chem. B. 2014; 118:1319213201. [PubMed: 25343221]

39. Szczelina R, Murzyn K. DMG-alpha--a computational geometry library for multimolecular systems. J. Chem. Inf. Model. 2014; 54:3112-3123. [PubMed: 25296168]

40. Abel S, Dupradeau F-Y, Marchi M. Molecular Dynamics Simulations of a Characteristic DPC Micelle in Water. J. Comput. Chem. 2012; 8:4610-4623.

41. Abel S, Lorieau A, de Foresta B, Dupradeau FY, Marchi M. Bindings of hMRP1 transmembrane peptides with dodecylphosphocholine and dodecyl-beta-d-maltoside micelles: a molecular dynamics simulation study. Biochim. Biophys. Acta. 2014; 1838:493-509. [PubMed: 24157718]

42. Hess B, Kutzner C, van der Spoel D, Lindahl E. Gromacs 4: Algorithms for highly efficient, loadbalanced, and scalable molecular simulation. J. Chem. Theory Comput. 2008; 4:435-447.

43. Tieleman DP, Maccallum JL, Ash WL, Kandt C, Xu Z, Monticelli L. Membrane protein simulations with a united-atom lipid and all-atom protein model: lipid-protein interactions, side chain transfer free energies and model proteins. J. Phys. Condens. Matter. 2006; 18:S1221-S1234. [PubMed: 21690838]

44. Armen RS, Uitto OD, Feller SE. Phospholipid component volumes: determination and application to bilayer structure calculations. Biophys. J. 1998; 75:734-744. [PubMed: 9675175]

45. Chiu SW, Pandit SA, Scott HL, Jakobsson E. An improved united atom force field for simulation of mixed lipid bilayers. J. Phys. Chem. B. 2009; 113:2748-2763. [PubMed: 19708111]

46. Petrache HI, Feller SE, Nagle JF. Determination of component volumes of lipid bilayers from simulations. Biophys. J. 1997; 72:2237-2242. [PubMed: 9129826]

47. Tieleman DP, Berendsen HJ. A molecular dynamics study of the pores formed by Escherichia coli OmpF porin in a fully hydrated palmitoyloleoylphosphatidylcholine bilayer. Biophys. J. 1998; 74:2786-2801. [PubMed: 9635733]

48. Tristram-Nagle S, Liu Y, Legleiter J, Nagle JF. Structure of gel phase DMPC determined by X-ray diffraction. Biophys. J. 2002; 83:3324-3335. [PubMed: 12496100]

49. Heerklotz H, Tsamaloukas A. Gradual change or phase transition: characterizing fluid lipidcholesterol membranes on the basis of thermal volume changes. Biophys. J. 2006; 91:600-607. [PubMed: 16632513]

50. Rothman JE, Engelman DM. Molecular mechanism for the interaction of phospholipid with cholesterol. Nat. New Biol. 1972; 237:42-44. [PubMed: 4503742]

51. $\mathrm{Xu} \mathrm{X}$, London E. The effect of sterol structure on membrane lipid domains reveals how cholesterol can induce lipid domain formation. Biochemistry. 2000; 39:843-849. [PubMed: 10653627]

52. de Meyer F, Smit B. Effect of cholesterol on the structure of a phospholipid bilayer. Proc. Natl. Acad. Sci. U.S.A. 2009; 106:3654-3658. [PubMed: 19225105]

53. Kukol A. Lipid models for united-atom molecular dynamics simulations of proteins. J. Chem. Theory Comput. 2009; 5:615-626. 


\section{Highlights}

- New 3D Voronoi Tessellation (VT) method to study protein-induced lipid disruption.

- Classification of multiple VT lipid shells based on VT nearest-atom-neighbors.

- Observed non-uniform recovery of fractional lipid volume across lipid shells.

- Bilayer thickness and lipid order parameter calculations validated the VT results.

- This method is applicable to all coordinate-based membrane structural data. 


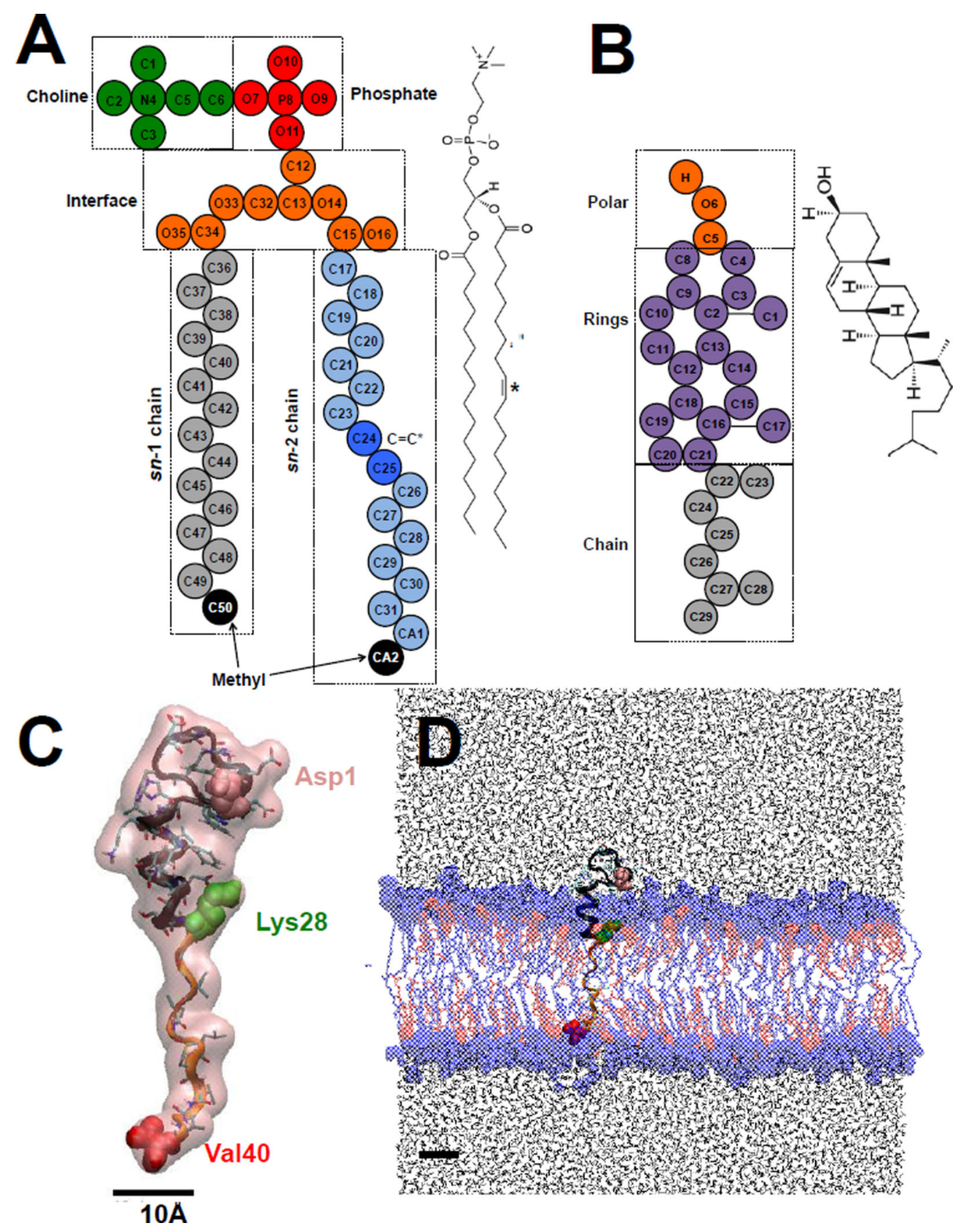

FIG. 1. Structures of lipid, protein, and protein/lipid bilayer in water

Color-coded atom types and chemical groups of PC (A) and CHOL (B) according to the published PDB files are shown. A transmembrane beta-amyloid (C) with backbone structure in ribbon, van der Waals surface in pink envelope and three residues (Asp1, Lys28, and Val40) in color spheres are given. A cross-section of the inserted beta-amyloid in a PC/ CHOL lipid bilayer in the presence of water (D) is illustrated. The polar headgroups of PC and CHOL are in blue and red spheres, respectively. The hydrophobic acyl chain tails of PC and CHOL are in blue and red lines, respectively. Water is in gray lines. Scale bar =10A. A 
comparison of the IUPAC-atom labeling and the PDB united atom labeling and a conversion Table are given in Supporting Material, Figure S1 and Table S1. 


\section{A}
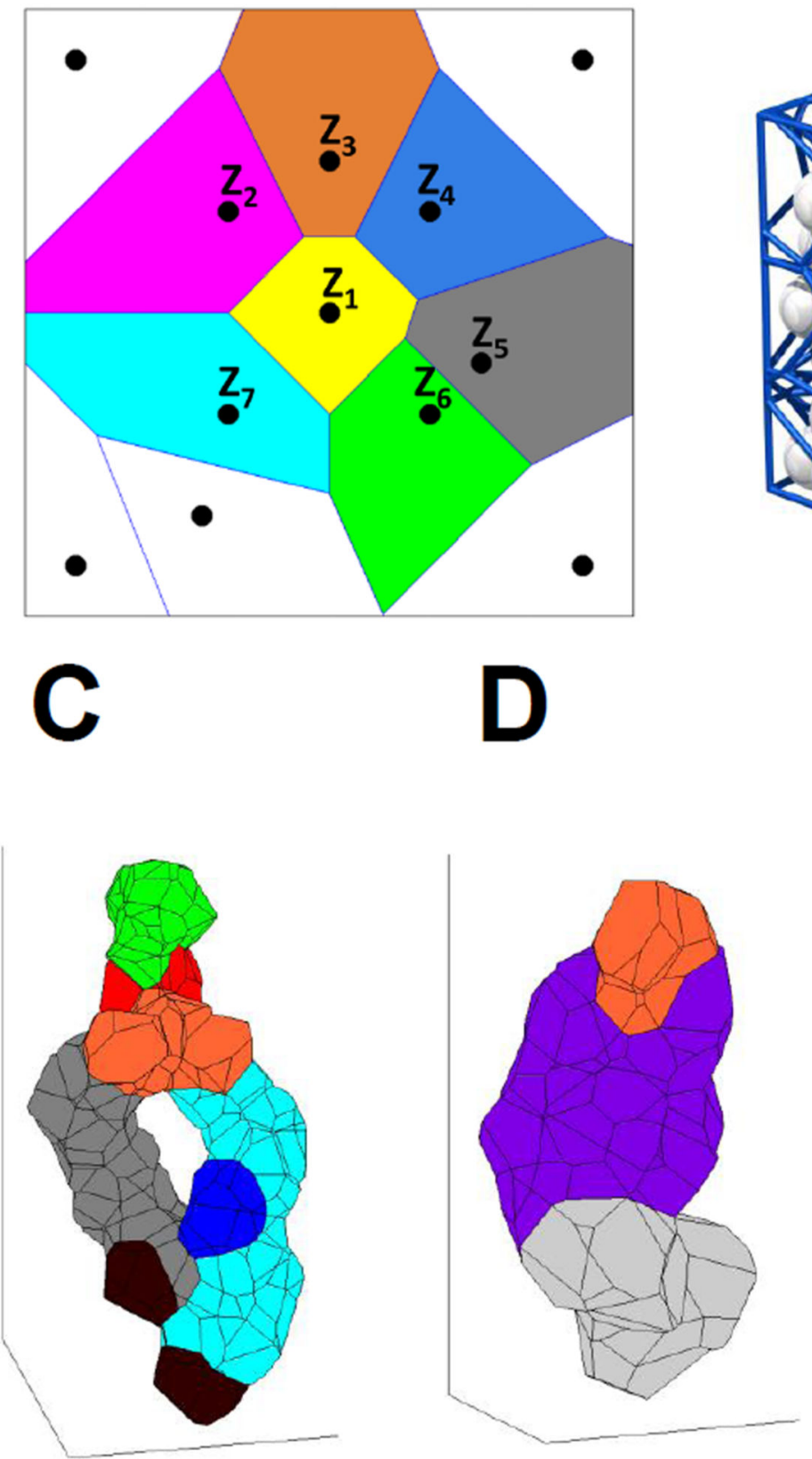

FIG. 2.
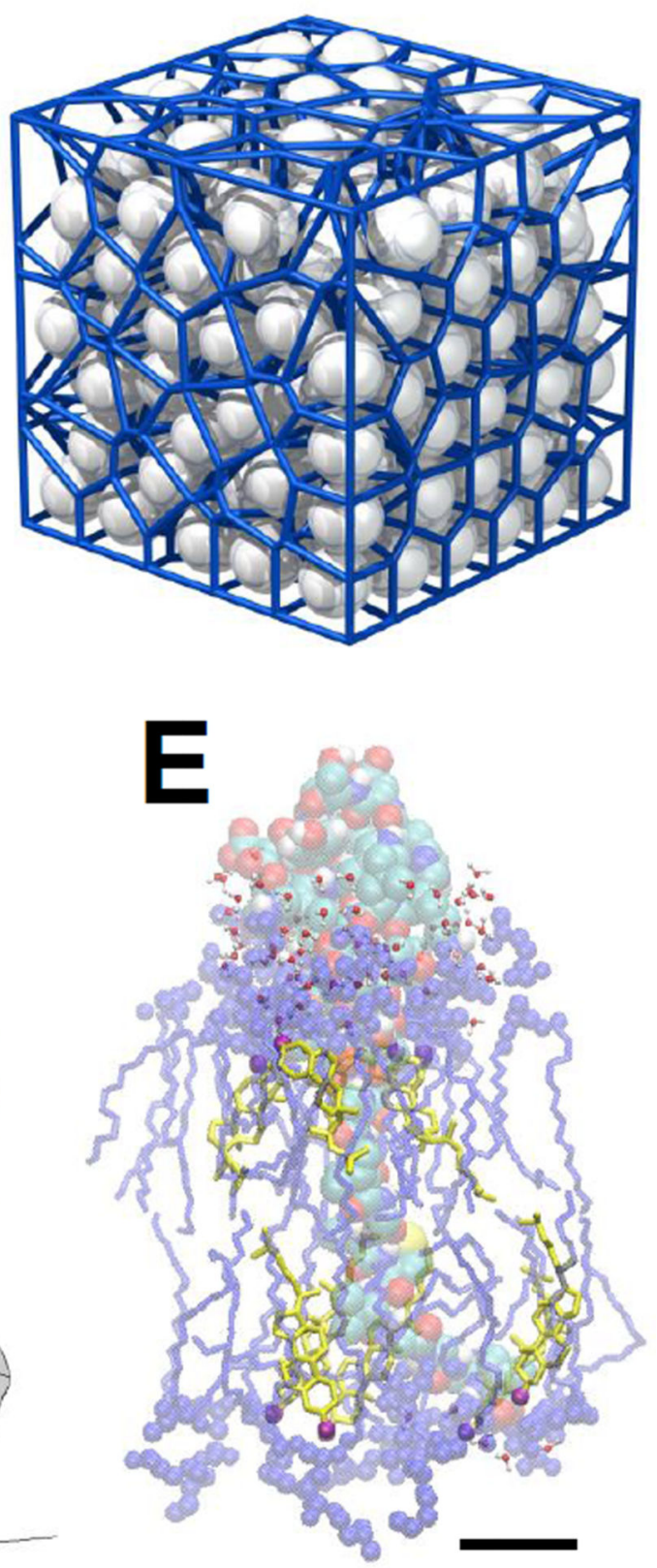

Demonstrations of multi-dimentional Voronoi tessellations. Division of 2D plane (A) and 3D cube (B) domains into Voronoi cells based on 12 random points and packed spheres, respectively. A 2D VT method divides a square domain $\Omega$ into twelve Voronoi polygons or $2 D$ cells. The generator $Z_{1}$ with its yellow Voronoi region has six nearest neighbors $\left(Z_{2}\right.$ to $\mathrm{Z}_{7}$ ) with different colored Voronoi regions. A 3D rendering of polyhedral volume occupied by each atom in a representative PC (C) and CHOL (D) molecule in a lipid bilayer. The colors correspond to those assigned to different atom group as given in Figure 1. Nearest 
neighbor lipids and water molecules to a beta-amyloid protein in a PC/CHOL bilayer (E) as determined from the nearest-atom-neighbor list of Voro++ are also given. The polar headgroups of PC and CHOL are represented by blue and red spheres, and the non-polar groups of $\mathrm{PC}$ and $\mathrm{CHOL}$ are represented by blue and yellow lines, respectively. The protein is shown by color spheres. Scale bar $=10 \AA$. See Materials and Methods for details. 


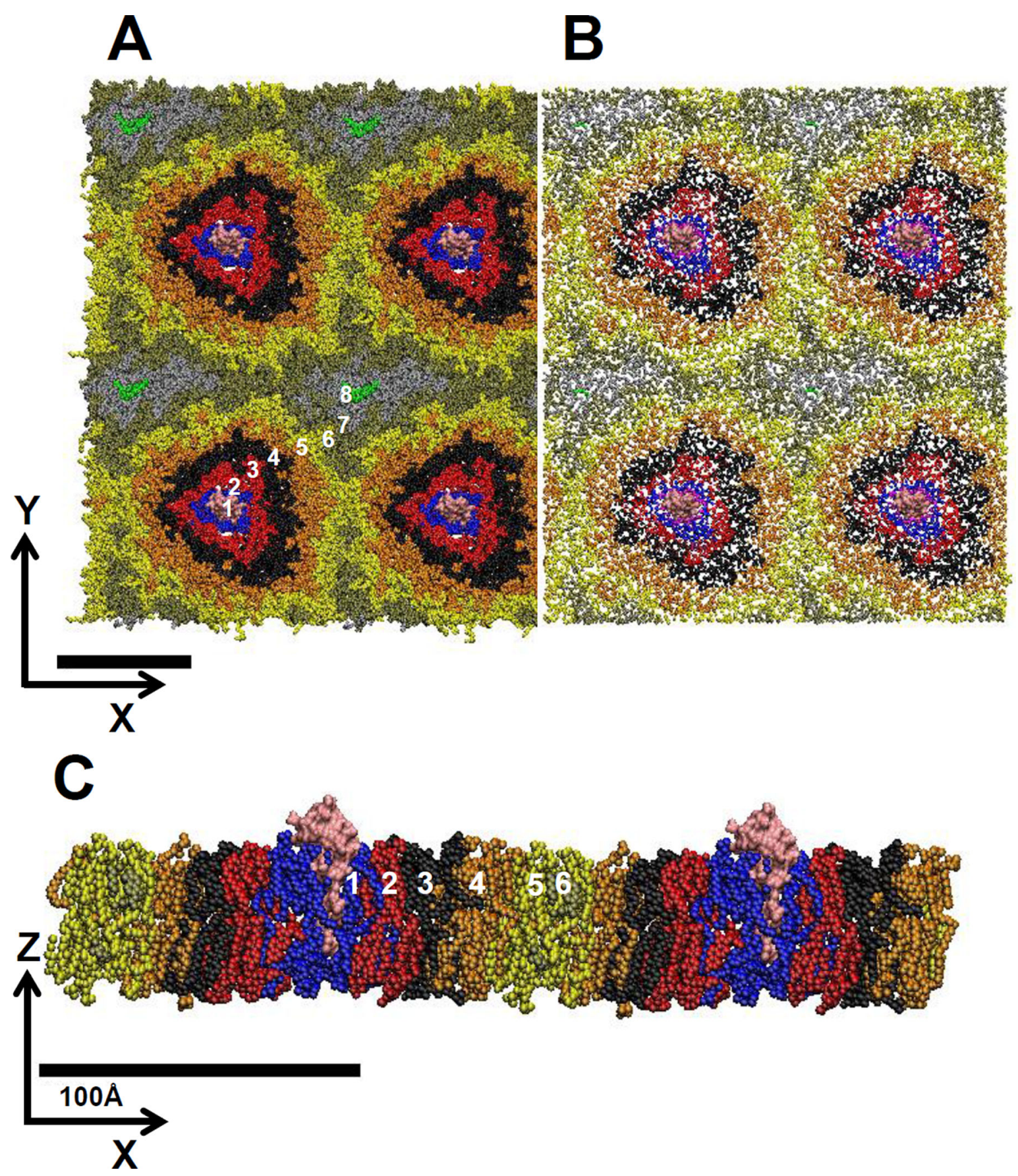

FIG. 3.

Classification of lipids and interfacial water in multiple VT lipid shells in a simulated protein/lipid bilayer system using weighted VT. Different lipids shells, in lateral (A) and transverse (C) views, in a simulated beta-amyloid/PC/CHOL bilayer are shown in blue, red, black, orange, yellow, dark green, gray, and light green according to shell order numbers from 1 to 8 , accordingly. The lipid-associated interfacial water shells (B) are shown with identical color codes. The interfacial water nearest to the protein $\left(0^{\text {th }}\right.$ order $)$ is marked in purple. The protein is shown in pink spheres. Periodic images of the system box along the $\mathrm{x}$ 
and $y$ directions are shown. The z-axis aligns with the normal of the bilayer. Scale bar $=100 \AA$. 

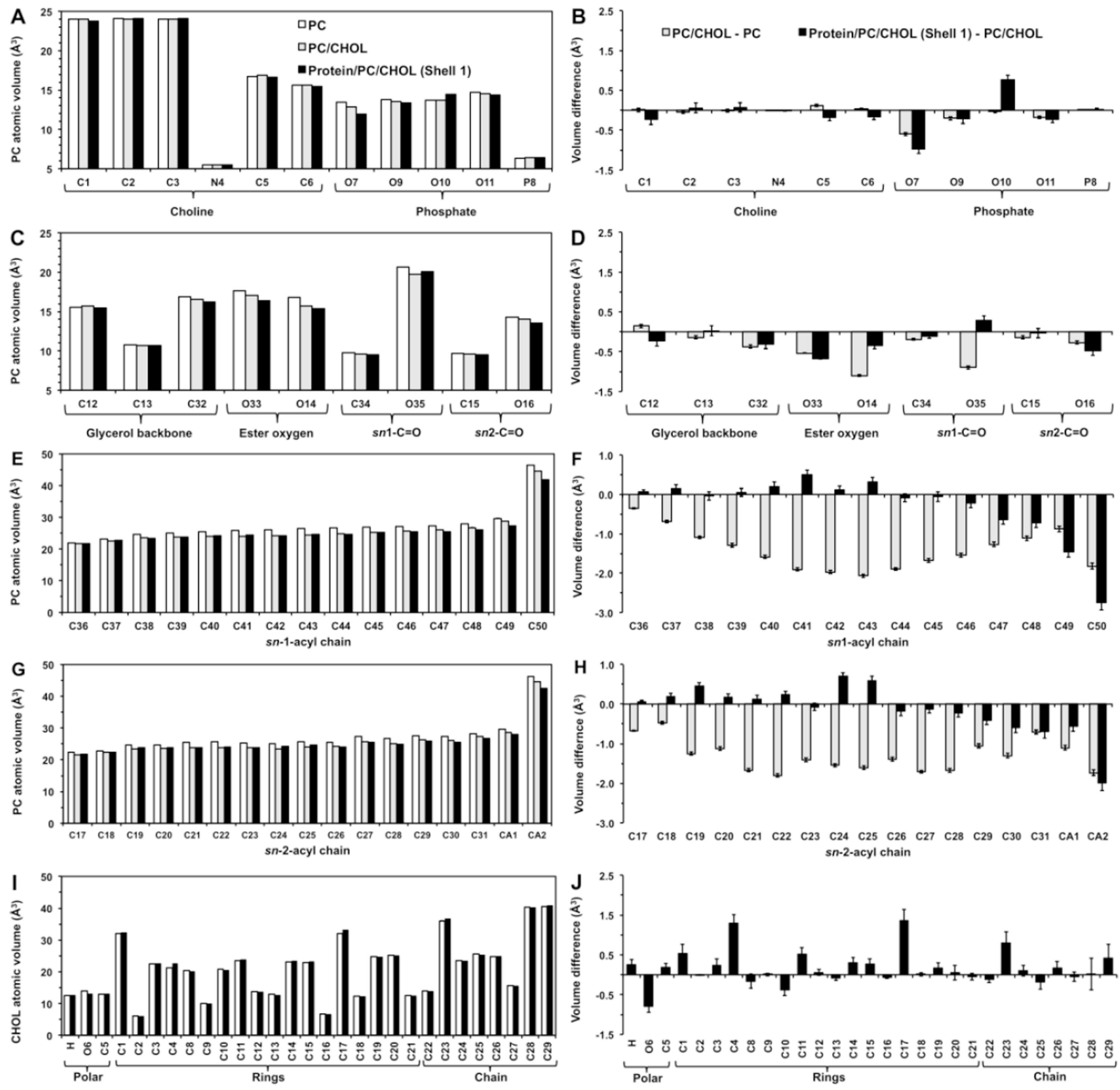

FIG. 4.

Profile of atomic volume of lipid in PC, PC/CHOL, and Protein/PC/CHOL bilayers (Shell 1) vs. atom type based on weighted VT method. The atomic volume of PC (A, C, E, and G) and CHOL (I) classified in different chemical groups as well as the corresponding volume difference (B, D, F, H, and J) are shown. Each data point represents an average over 30 frames (170 to $200 \mathrm{~ns}$ with $1 \mathrm{~ns}$ increment) and the error bar indicates the standard error of the mean. 
PC-headgroup
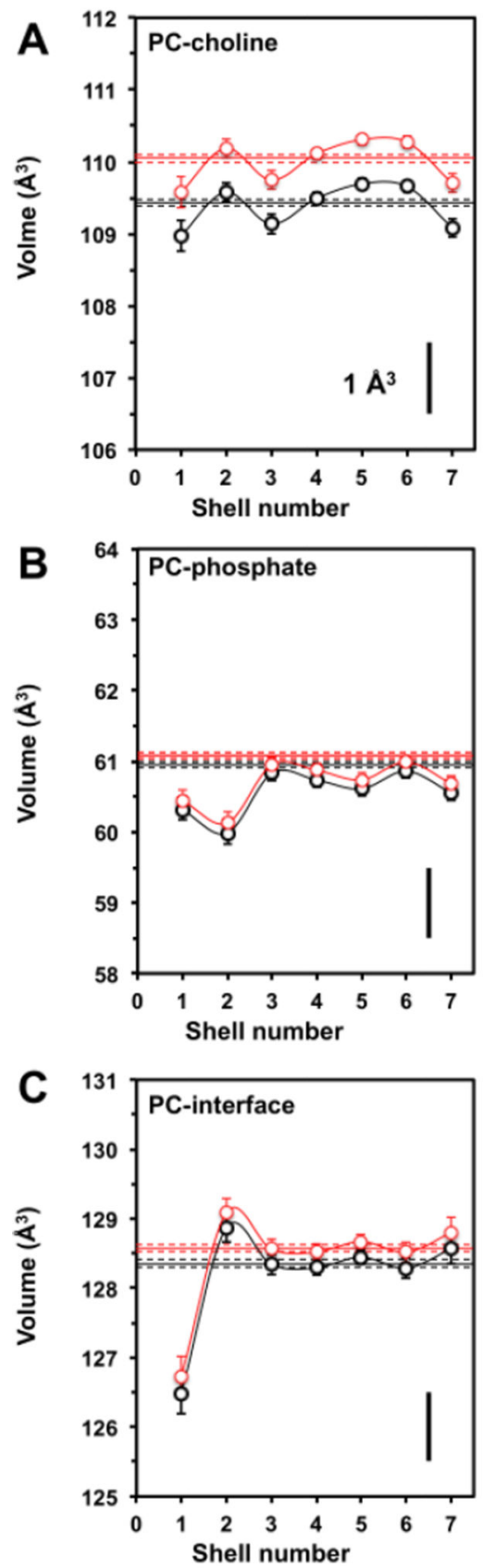

PC-tails
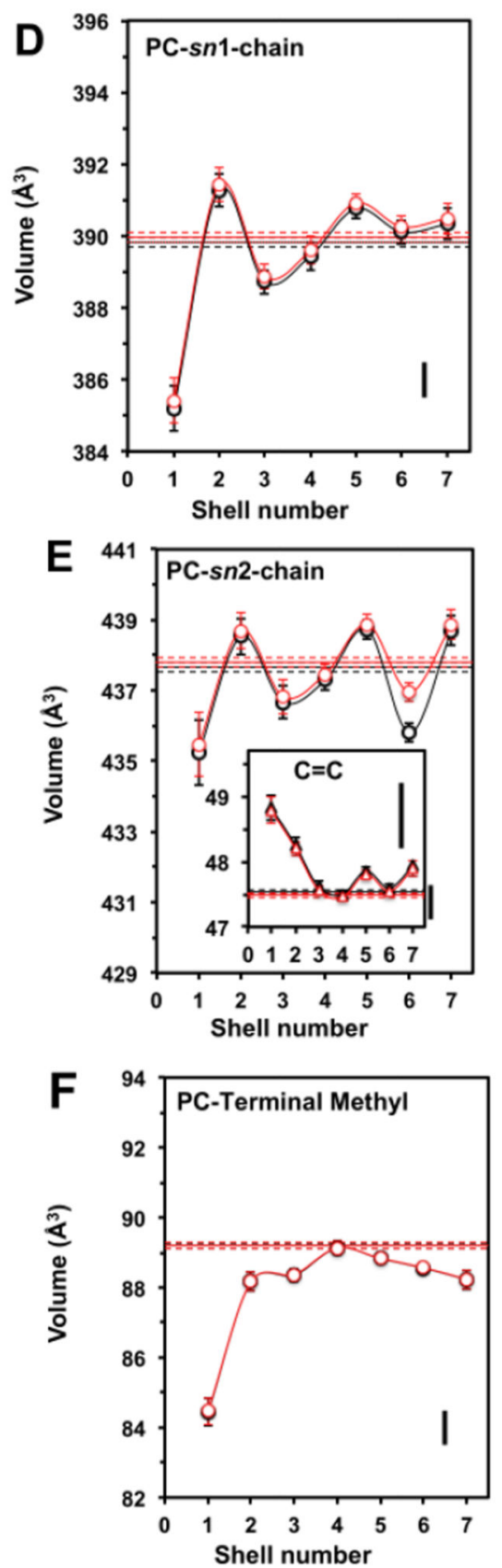

CHOL
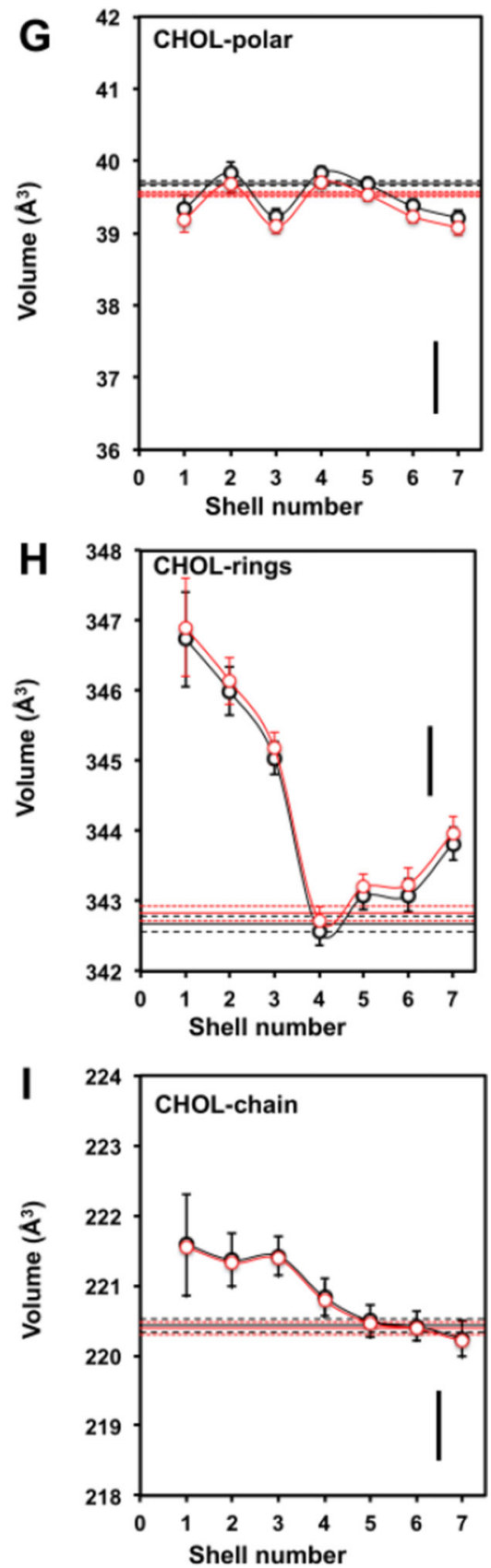

FIG. 5.

Fractional volume of lipid vs. VT lipid shell order number. The average volumes of selected atom groups: choline (A), phosphate $(\mathrm{B})$, interface $(\mathrm{C})$, acyl chains $(\mathrm{D}$ and $\mathrm{E}), \mathrm{C}=\mathrm{C}(\mathrm{E}$ insert) and terminal $\mathrm{CH}_{3}(\mathrm{~F})$ of $\mathrm{PC}$, as well as the polar $(\mathrm{G})$, rings $(\mathrm{H})$ and chain (I) of CHOL are shown for weighted (red) and non-weighted VT (black). To facilitate cross comparisons, a thick vertical bar of $1 \AA^{3}$ is given. See the legend of Figure 4 for details in the uncertainties of data points. The corresponding values of the control, i.e., $\mathrm{PC} / \mathrm{CHOL}$ with no 
protein, are given by horizontal lines with dotted lines highlight the standard errors of the means. 

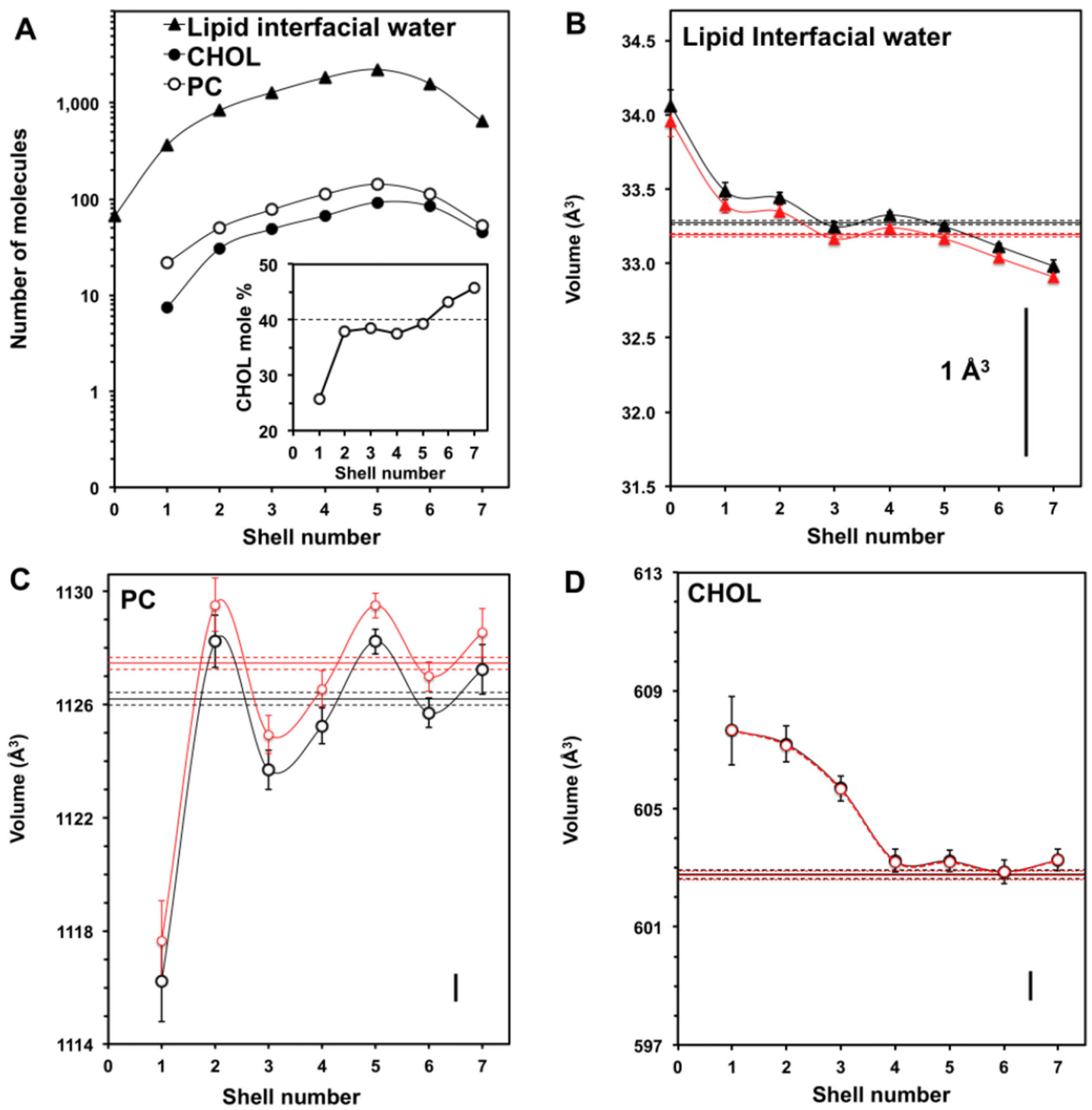

FIG. 6.

Molecular volume of lipid and interfacial water vs. VT shell order number. The lipid and water concentration (A), volume of interfacial water (B), volume of PC (C) and volume of CHOL (D) in each VT shell are shown for weighted VT (red) and non-weighted VT (black).

The corresponding values of the control, i.e., PC/CHOL with no protein, are given by horizontal lines with dotted lines highlight the standard errors of the means. See the legend of Figure 4 for details in the uncertainties of data points. 

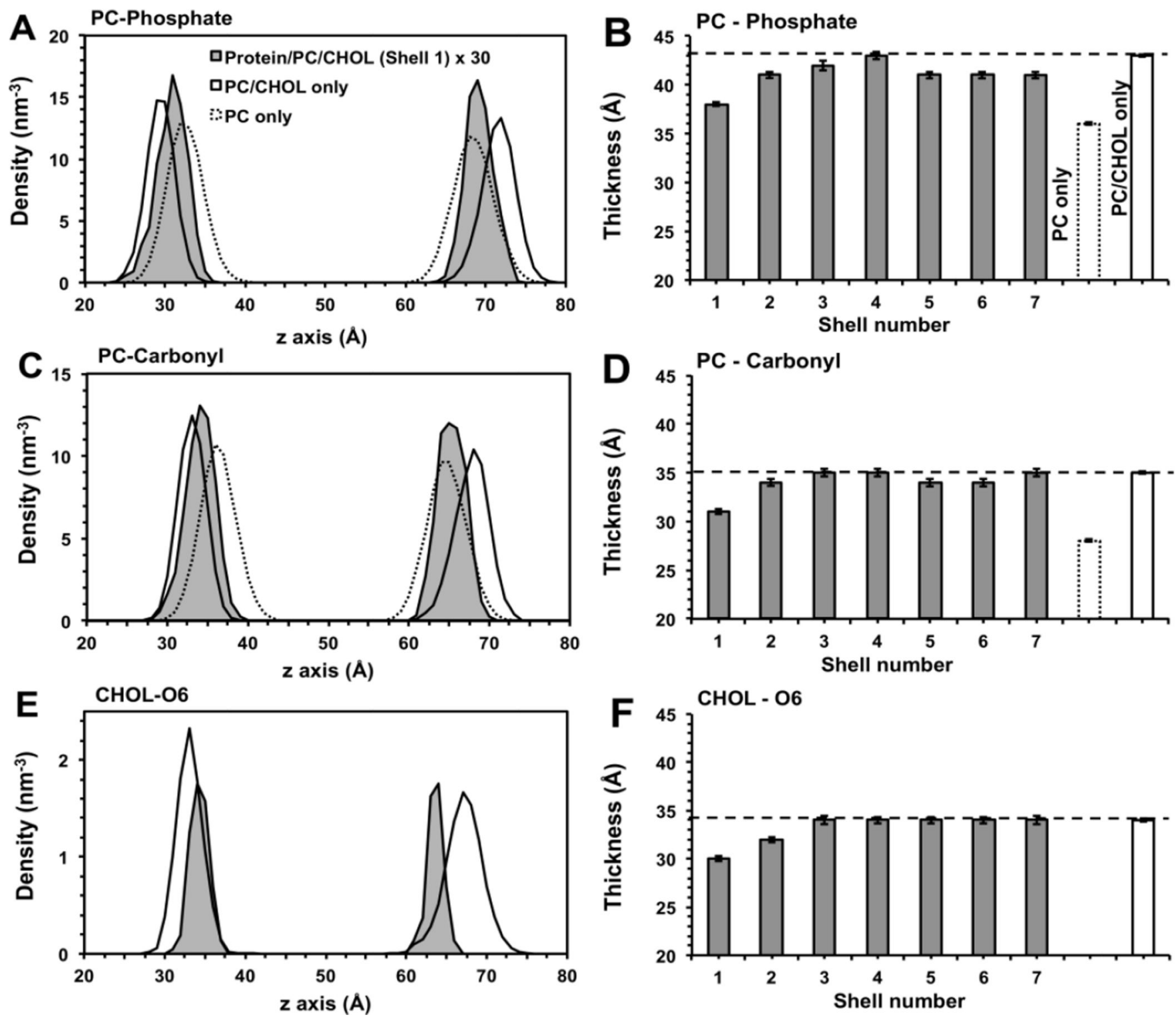

FIG. 7.

Number density of lipid polar group vs. VT lipid shell order number. Plots of number density vs. z-axis of the phosphate (A) and carbonyl (C) groups of PC and the O6 (E) group of CHOL of the protein/PC/CHOL bilayer are shown using weighted VT. Plots of the average polar group thickness defined as peak-to-peak z-distance of the corresponding polar groups (B, D and F) are also shown. Plots of the average thickness of the PC and PC/CHOL bilayers in the absence of protein (controls) are also shown. See the legend of Figure 4 for details in the uncertainties of data points. 

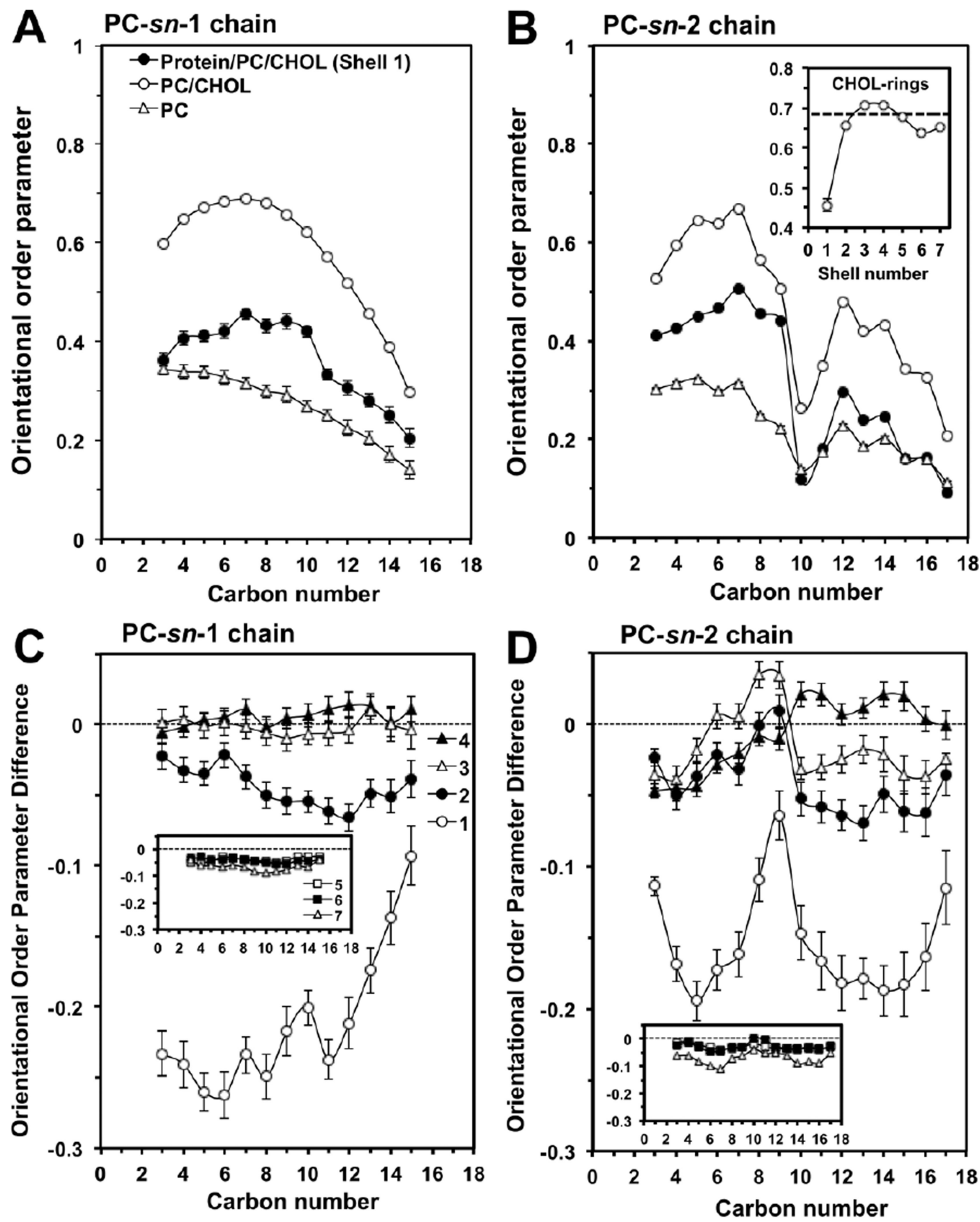

FIG. 8.

Lipid orientational order in lipid shells. The orientational order of PC acyl chain vs. chain carbon number in PC, PC/CHOL and protein/PC/CHOL bilayers (Shell 1) for the saturated $s n-1$ chain (A) and unsaturated $s n-2$ chain $(\mathrm{B})$ are shown using weighted VT. The corresponding order parameter difference (= order parameter in the protein/PC/CHO bilayer minus that in the control PC/CHOL bilayer) plots ( $\mathrm{C}$ and $\mathrm{D}$ ) are also presented. For clarity, the volume difference of the higher order lipid shells are shown in the inserts. The order 
parameter of the rings of the $\mathrm{CHOL}$ is also shown (insert of panel B). See the legend of Figure 4 for details in the uncertainties of data points. 